\title{
A PHASE SPACE TRANSFORM ADAPTED TO THE WAVE EQUATION
}

\author{
DAN-ANDREI GEBA AND DANIEL TATARU
}

\begin{abstract}
Wave packets emerged in recent years as a very useful tool in the study of nonlinear wave equations. In this article we introduce a phase space transform adapted to the geometry of wave packets, and use it to characterize and study the associated classes of pseudodifferential and Fourier integral operators.
\end{abstract}

\section{INTRODUCTION}

A natural way to study pseudodifferential and Fourier integral operators is by means of phase space transforms. This is easiest to understand within the framework of the $S_{00}^{0}$ calculus, where the localization occurs on the unit scale both in position and in the frequency. The corresponding phase space transform is precisely the Bargmann transform,

$$
T u(x, \xi)=c_{n} \int_{\mathbb{R}^{n}} e^{i \xi(x-y)} e^{-\frac{(x-y)^{2}}{2}} u(y) d y
$$

The Bargmann transform is an isometry from $L^{2}\left(\mathbb{R}^{n}\right)$ into $L^{2}\left(\mathbb{C}^{n}\right)$ so an inverse for it is provided by the adjoint operator. This inverse is not uniquely determined since $T$ is not onto. Precisely, the range of $T$ consists of those functions satisfying a Cauchy-Riemann type equation, $i \partial_{\xi} v=\left(\partial_{x}-i \xi\right) v$. The connection with the $S_{00}^{0}$ type calculus is provided by the following simple result:

Theorem 1.1. ([14]) Let $A: S\left(\mathbb{R}^{n}\right) \rightarrow S^{\prime}\left(\mathbb{R}^{n}\right)$ be a linear operator. Then $A \in O P S_{00}^{0}$ if and only if the kernel $K$ of $T A T^{*}$ satisfies

$$
\left|K\left(x_{1}, \xi_{1}, x_{2}, \xi_{2}\right)\right| \leq c_{N}\left(1+\left|x_{1}-x_{2}\right|+\left|\xi_{1}-\xi_{2}\right|\right)^{-N} \quad \forall N \in \mathbb{N} .
$$

This provides an easy way to study the calculus and the $L^{2}$ boundedness of $O P S_{00}^{0}$ pseudodifferential operators. One can also talk about $S_{00}^{0}$ type Fourier integral operators, etc. For more details and further development of these ideas we refer the reader to [5], [6], [15], [14].

On the other hand, in the study of the wave equation with rough coefficients one is naturally led to consider wave packets. These are

Date: August 22, 2005. 
exact or approximate solutions to the wave equation which are localized in position and frequency on dual scales. Wave packets were first introduced in the work of Smith [12], [11], and since then have found many uses both theoretically (see [16], [13], [17]) and numerically (see [3] and references therein).

In the initial data space, the wave packets correspond to what is called the second dyadic decomposition. Precisely, we begin with a dyadic decomposition in frequency; then, each dyadic annulus of size $\lambda$ is subdivided into sectors of angle $\lambda^{-\frac{1}{2}}$. Thus the Fourier space is partitioned into boxes which at frequency $\lambda$ have size $\lambda \times\left(\lambda^{\frac{1}{2}}\right)^{n-1}$. Then for each such box one considers an equipartition of the physical space into boxes on the dual scale $\lambda^{-1} \times\left(\lambda^{-\frac{1}{2}}\right)^{n-1}$.

One can decompose any initial data set for the wave equation into a discrete, almost orthogonal superposition of localized initial data on the above scale. Then the wave packets are essentially obtained by transporting those initial data along the corresponding Hamilton flow.

The first aim of this paper is to introduce a phase space transform adapted to the scales described above. Via an inversion formula this leads to a continuous (even smooth) counterpart of the discrete second dyadic decomposition for the initial data.

Then we consider the associated classes of symbols, and characterize the corresponding pseudodifferential operators using our phase space transform. This analysis is not entirely straightforward as it shares some of the features of the $S_{1,1}$ calculus.

Starting with a suitable class of canonical transformations we introduce the Fourier integral operators adapted to this geometry. For these we discuss the calculus and the $L^{2}$ boundedness properties.

Finally, we consider evolution equations governed by first order operators with almost homogeneous symbols, and we show that the generated evolution operators are in effect Fourier integral operators associated to the canonical transformations generated by the Hamilton flow. In the case of $S_{00}^{0}$ calculus this analysis was carried out in [10], [14]. For similar results see also [2].

As an application, we consider the question of constructing parametrices for half-wave evolutions with rough coefficients. In the spirit of the paradifferential calculus, we regularize the coefficients on a frequency dependent scale to obtain a modified evolution which fits within our setup. On the other hand we show that the original and the modified evolutions are close in the $L^{2}$ sense. 


\section{The phase space structure}

Given two vectors $\xi, \eta \in \mathbb{R}^{n}$ we denote

$$
\xi \wedge \eta=\xi \otimes \eta-\eta \otimes \xi
$$

We can decompose $\eta$ into components which are parallel, respectively perpendicular to $\xi$ by

$$
\eta=\frac{1}{\xi^{2}}((\eta \cdot \xi) \xi+(\eta \wedge \xi) \xi)
$$

The Euclidean operator norm of $\xi \wedge \eta$ is denoted by $|\xi \wedge \eta|$. One can show that it equals exactly $|\xi|$ times the length of the component of $\eta$ which is perpendicular to $\xi$.

In the Fourier space we define a Riemannian metric $g$, which at the point $\xi$ is defined by

$$
g_{\xi}(\eta)=\frac{1}{\xi^{2}\left(1+\xi^{2}\right)}(\xi \cdot \eta)^{2}+\frac{1}{\xi^{2}\left(1+\xi^{2}\right)^{\frac{1}{2}}}|\xi \wedge \eta|^{2}
$$

In the physical space we use a dual scale, given by the metric $g^{-1}$. Consequently we set

$$
g_{\xi}^{-1}(y)=\frac{1+\xi^{2}}{\xi^{2}}(\xi \cdot y)^{2}+\frac{\left(1+\xi^{2}\right)^{\frac{1}{2}}}{\xi^{2}}|\xi \wedge y|^{2}
$$

Putting these two together we obtain a Riemannian metric in $T^{*} \mathbb{R}^{n}$, given by

$$
d s^{2}=g_{\xi}(d \xi)+g_{\xi}^{-1}(d x)
$$

By a slight abuse of notation we also use $g$ for the phase space metric and set

$$
g_{x, \xi}(y, \eta)=g_{\xi}^{-1}(y)+g_{\xi}(\eta)
$$

We denote by $d$ the distance induced by this metric in $T^{*} \mathbb{R}^{n}$. On occasion we omit the spatial component,

$$
d(\xi, \eta):=d((0, \xi),(0, \eta))
$$

For $\xi \in \mathbb{R}^{n}$ we denote a unit size neighborhood by

$$
R_{\xi}=\left\{\eta \in \mathbb{R}^{n} ; g_{\xi}(\xi-\eta) \leq 1 / 4\right\}
$$

This is a dyadic sector around $\xi$ of radial length $\langle\xi\rangle$ and width $\langle\xi\rangle^{\frac{1}{2}}$ (or equivalently, of angle $\langle\xi\rangle^{-\frac{1}{2}}$ ). It is easy to verify that $g$ is a slowly varying metric, in the sense that

Lemma 2.1. The metric $g$ is slowly varying. Precisely,

$$
\xi \in R_{\eta} \Longrightarrow \frac{1}{16} g_{\eta} \leq g_{\xi} \leq 16 g_{\eta}
$$


This shows that within $R_{\xi}$ we can freeze the metric to $g_{\xi}$ and obtain an equivalent distance. Thus $R_{\xi}$ roughly coincides with a unit sized ball with respect to the metric $g$.

The dual balls to $R_{\xi}$ in the physical space are denoted by

$$
R_{\xi}^{-1}=\left\{y \in \mathbb{R}^{n} ; g_{\xi}^{-1}(y) \leq 4\right\}
$$

These are roughly boxes of size $\langle\xi\rangle^{-1}$ in the $\xi$ direction and $\langle\xi\rangle^{-\frac{1}{2}}$ in all directions normal to $\xi$. Then we can describe small balls in the phase space roughly by

$$
B((x, \xi), \epsilon) \approx\left(x+\epsilon R_{\xi}^{-1}\right) \times \epsilon R_{\xi}, \quad \epsilon \leq 1
$$

To measure the regularity of functions on the scale given by the metric $g$ we use the notation

$$
\left|\nabla^{k} a(x, \xi)\right|_{g}=\sup \left\{\left(\Pi_{j=1}^{k} v_{j} \nabla\right) a(x, \xi) ; g_{x, \xi}\left(v_{j}\right) \leq 1, j=\overline{1, k}\right\}
$$

Since $g$ is slowly varying, within unit size balls we can freeze the metric for this purpose. We can control the regularity of the metric $g$ on the $g$ scale:

Lemma 2.2. The metric $g$ is smooth on the $g$ scale,

$$
\left|\nabla_{\xi}^{\alpha} g_{\xi}(\eta)\right|_{g} \lesssim g_{\xi}(\eta), \quad \eta \in \mathbb{R}^{n}
$$

Proof. We can write $g_{\xi}$ as a matrix,

$$
g_{\xi}=\frac{1}{1+\xi^{2}} \frac{\xi}{|\xi|} \otimes \frac{\xi}{|\xi|}+\frac{1}{\left(1+\xi^{2}\right)^{\frac{1}{2}}}\left(I_{n}-\frac{\xi}{|\xi|} \otimes \frac{\xi}{|\xi|}\right)
$$

When $|\xi|<1$ we only need to verify that all components are smooth, which is easy. For larger $\xi$ we can assume that $\xi=r e_{1}$. We rescale

$$
\xi_{1}=r \zeta_{1}, \quad \xi^{\prime}=r^{\frac{1}{2}} \zeta^{\prime}
$$

to bring $\xi$ to $e_{1}$ and $g_{\xi}$ to $I_{n}$. The metric $g$ in the new coordinates has the form

$$
\begin{aligned}
g_{r}(\zeta)= & \frac{\left(\zeta_{1}, r^{-\frac{1}{2}} \zeta^{\prime}\right) \otimes\left(\zeta_{1}, r^{-\frac{1}{2}} \zeta^{\prime}\right)}{\left(r^{-2}+\zeta_{1}^{2}+r^{-1} \zeta^{\prime 2}\right)\left(\zeta_{1}^{2}+r^{-1} \zeta^{\prime 2}\right)} \\
& +\frac{\zeta^{\prime 2} e_{1} \otimes e_{1}-\zeta_{1}\left(e_{1} \otimes\left(0, \zeta^{\prime}\right)+\left(0, \zeta^{\prime}\right) \otimes e_{1}\right)}{\left(r^{-2}+\zeta_{1}^{2}+r^{-1} \zeta^{\prime 2}\right)^{\frac{1}{2}}\left(\zeta_{1}^{2}+r^{-1} \zeta^{\prime 2}\right)} \\
& +\frac{\left(\zeta_{1}^{2}+r^{-1} \zeta^{\prime 2}\right) I_{n-1}^{\prime}-\left(0, \zeta^{\prime}\right) \otimes\left(0, \zeta^{\prime}\right)}{\left(r^{-2}+\zeta_{1}^{2}+r^{-1} \zeta^{\prime 2}\right)^{\frac{1}{2}}\left(\zeta_{1}^{2}+r^{-1} \zeta^{\prime 2}\right)}
\end{aligned}
$$


In the rescaled setting we need to prove that the derivatives of the components of $g_{r}$ at $\zeta=e_{1}$ are uniformly bounded with respect to $r \geq 1$. But this is straightforward.

The above results show that the short range geometry is essentially flat, and is described by the frozen metric $g_{\xi}$. This is no longer the case for the long range geometry. To characterize it we begin with a simpler result allowing us to compare $g$ at different points:

Lemma 2.3. Let $\xi, \eta \in \mathbb{R}^{n}$. Then

$$
g_{\xi}^{-1}(u) \lesssim g_{\eta}^{-1}(u)\left(1+g_{\eta}(\xi-\eta)\right)
$$

and the dual bound

$$
g_{\xi}(u) \lesssim g_{\eta}(u)\left(1+g_{\xi}(\xi-\eta)\right)
$$

Proof. Since $g_{\eta}(\eta) \leq 1$ it follows that

$$
\left(1+g_{\eta}(\xi-\eta)\right) \approx 1+g_{\eta}(\xi)
$$

We write $\xi$ as

$$
\xi=\frac{\xi \cdot \eta}{\eta^{2}} \eta+\frac{1}{\eta^{2}}(\xi \wedge \eta) \eta
$$

Then we express the component of $u$ along $\xi$ as

$$
\xi \cdot u=\frac{\xi \cdot \eta}{\eta^{2}} \eta \cdot u+\frac{1}{\eta^{2}}(\xi \wedge \eta) \eta \cdot u
$$

Hence we have

$$
\begin{aligned}
g_{\xi}^{-1}(u) & \lesssim \frac{\langle\xi\rangle^{2}}{\xi^{2} \eta^{4}}\left(|\xi \cdot \eta|^{2}|\eta \cdot u|^{2}+|\xi \wedge \eta|^{2}|u|^{2}|\eta|^{2}\right)+\langle\xi\rangle|u|^{2} \\
& \lesssim g_{\eta}^{-1}(u)\left(\frac{\langle\xi\rangle^{2}}{\langle\eta\rangle^{2}}+\frac{\langle\xi\rangle^{2}|\xi \wedge \eta|^{2}}{|\xi|^{2}|\eta|^{2}\langle\eta\rangle}+\frac{\langle\xi\rangle}{\langle\eta\rangle}\right) \\
& \lesssim g_{\eta}^{-1}(u)\left(1+\frac{\xi^{2}}{\langle\eta\rangle^{2}}+\frac{|\xi \wedge \eta|^{2}}{|\eta|^{2}\langle\eta\rangle}\right) \\
& \approx g_{\eta}^{-1}(u)\left(1+g_{\eta}(\xi)\right)
\end{aligned}
$$

This allows us to consider the size of $g$ at distant points.

Lemma 2.4. Let $\xi_{1}, \xi_{2} \in \mathbb{R}^{n}$. Then for $\eta \in R_{\xi_{1}}$ and $\xi \in R_{\xi_{2}}$ we have

$$
1+g_{\xi_{1}}(\eta-\xi) \approx 1+g_{\xi_{1}}\left(\xi_{1}-\xi_{2}\right)
$$


Proof. We first note that $g_{\xi_{1}}\left(\eta-\xi_{1}\right) \approx 1$, so without any restriction in generality we can take $\eta=\xi_{1}$. By Lemma 2.3 we also have

$$
g_{\xi_{1}}\left(\xi-\xi_{2}\right) \lesssim g_{\xi_{2}}\left(\xi-\xi_{1}\right)\left(1+g_{\xi_{1}}\left(\xi_{1}-\xi_{2}\right)\right) \lesssim\left(1+g_{\xi_{1}}\left(\xi_{1}-\xi_{2}\right)\right)
$$

Then the "¿" bound follows. The opposite inequality must also be true by symmetry.

Finally, we give a complete characterization of the distance $d$.

Theorem 2.5. We have

$$
1+d(\xi, \eta) \approx \ln \left(g_{\xi}(\xi-\eta)+\frac{\langle\xi\rangle}{\langle\eta\rangle}+\frac{\langle\eta\rangle}{\langle\xi\rangle}+\langle\xi, \eta\rangle_{-}\right)
$$

and

(2) $1+d((x, \xi),(y, \eta)) \approx 1+d(\xi, \eta)+\ln \left(1+g_{\xi}^{-1}(x-y)\right)+|x-y|$

We note that the role of the last term in (1) is roughly to differentiate the region where $\eta$ is close to $\xi$ from the region where $\eta$ is close to $-\xi$.

Proof. For (1), we first find a path between $\xi$ and $\eta$ whose length is comparable to the right hand side. The intuitive idea is that it is less costly to move in angle at a lower speed. Therefore we choose the following route $\gamma_{0}$ :

- from $\xi$ and $\eta$ we go radially towards the origin until the vectors are in the same dyadic angular piece centered at another vector $\zeta_{0}$;

- then we move in angle inside this piece.

To estimate the length of this path we express the metric in polar coordinates

$$
\xi=r \Theta
$$

Then

$$
d s^{2}=(d \ln \langle r\rangle)^{2}+\langle r\rangle^{-1}(d \Theta)^{2}
$$

Hence the length of $\gamma_{0}$ is

$$
1+l\left(\gamma_{0}\right) \approx 1+\ln \frac{\langle\xi\rangle}{\left\langle\zeta_{0}\right\rangle}+\ln \frac{\langle\eta\rangle}{\left\langle\zeta_{0}\right\rangle}=1+\ln \frac{\langle\xi\rangle\langle\eta\rangle}{\left\langle\zeta_{0}\right\rangle^{2}}
$$

To determine $\left\langle\zeta_{0}\right\rangle$ we assume that $\langle\xi\rangle \geq\langle\eta\rangle$ and consider two cases depending on the size of the angle $\theta$ between $\xi$ and $\eta$.

Case $1, \theta<\langle\eta\rangle^{-\frac{1}{2}}$. Then we take $\zeta_{0}=\eta$ therefore

$$
1+l\left(\gamma_{0}\right) \approx 1+\ln \frac{\langle\xi\rangle}{\langle\eta\rangle}
$$


This is comparable to the right hand side of (1) since

$$
g_{\xi}(\xi-\eta)+\frac{\langle\eta\rangle}{\langle\xi\rangle} \lesssim 1
$$

while the last term $\langle\xi, \eta\rangle_{-}$is 0 if $\langle\eta\rangle \gg 1$ and $O(\langle\xi\rangle)$ otherwise.

Case $2, \theta \geq\langle\eta\rangle^{-\frac{1}{2}}$. Then $\theta \approx\left\langle\zeta_{0}\right\rangle^{-\frac{1}{2}}$ therefore

$$
1+l\left(\gamma_{0}\right) \approx 1+\ln \frac{\langle\xi\rangle}{\langle\eta\rangle}+\ln \left(\langle\eta\rangle^{2} \theta^{4}\right)
$$

On the other hand in the right hand side of (1) we have

$$
1+g_{\xi}(\xi-\eta) \approx 1+\frac{\eta^{2} \sin ^{2} \theta}{\langle\xi\rangle} \approx 1+\frac{\langle\eta\rangle}{\langle\xi\rangle}\left(\langle\eta\rangle \sin ^{2} \theta\right)
$$

while

$$
\langle\xi, \eta\rangle_{-}=|\xi||\eta|(\cos \theta)_{-} \lesssim \frac{\langle\xi\rangle}{\langle\eta\rangle}\left(\langle\eta\rangle^{2} \theta^{4}\right)
$$

Clearly we get

$$
1+l\left(\gamma_{0}\right) \gtrsim R H S(1)
$$

For the converse we consider two cases. If $\theta<3 \pi / 4$ then $\sin \theta \approx \theta$ so we can use the $g_{\xi}(\xi-\eta)$ term to control $l(\gamma)$. Otherwise $(\cos \theta)_{-} \approx 1$, so we use the $\langle\xi, \eta\rangle_{-}$term.

To complete the proof of (1) it remains to show that the length of any path $\gamma$ joining $\xi$ and $\eta$ has $\operatorname{RHS}(1)$ as a lower bound. We have

$$
l(\gamma) \approx \int\left(g_{\gamma(t)}(\dot{\gamma}(t))\right)^{\frac{1}{2}} d t
$$

Choose $\zeta$ on $\gamma$ of minimum norm, $|\zeta|=\min |\gamma(t)|$. Using the expression (3) of the metric in polar coordinates we can infer immediately that:

$$
l(\gamma) \gtrsim \ln \frac{\langle\xi\rangle\langle\eta\rangle}{\langle\zeta\rangle^{2}}+\theta\langle\zeta\rangle^{\frac{1}{2}}=f(\langle\zeta\rangle)
$$

Given the choice of $\zeta_{0}$ above, it remains to show that $\zeta_{0}$ is an approximate minimum for $f$,

$$
f(\langle\zeta\rangle) \gtrsim f\left(\left\langle\zeta_{0}\right\rangle\right), \quad\langle\zeta\rangle \leq\langle\eta\rangle \leq\langle\xi\rangle
$$

But this is a straightforward computation.

We now turn our attention to the investigation of (2). First we make the observation that

$$
d((x, \xi),(y, \eta)) \approx d((x, \xi),(y, \xi))+d(\xi, \eta)
$$

This is immediate using the triangle inequality and

$$
d((x, \xi),(y, \eta)) \geq d(\xi, \eta)=d((y, \xi),(y, \eta))=d((x, \xi),(x, \eta))
$$


This reduces the proof of (2) to the case when $\xi=\eta$. We proceed as in the first part. First we construct a geodesic whose length is comparable to $\ln \left(1+g_{\xi}^{-1}(x-y)\right)+|x-y|$.

We consider the piecewise straight trajectory

$$
\gamma(\zeta):(x, \xi) \rightarrow(x, \zeta) \rightarrow(y, \zeta) \rightarrow(y, \xi)
$$

where $\zeta \in[0, \xi]$. This has length

$$
l(\gamma(\zeta)) \approx \sqrt{g_{\zeta}^{-1}(x-y)}+\ln \frac{\langle\xi\rangle}{\langle\zeta\rangle}=f(\zeta)
$$

Then we optimize its length with respect to $\zeta \in[0, \xi]$ and show that

$$
\min _{\lambda \in[0,1]} f(\lambda) \approx \ln \left(1+g_{\xi}^{-1}(x-y)\right)+|x-y|
$$

For one direction we note the trivial bound $f(\lambda) \geq|x-y|$. In addition,

$$
f(\lambda) \gtrsim \ln \left(1+g_{\zeta}^{-1}(x-y)\right)+\ln \frac{\langle\xi\rangle}{\langle\zeta\rangle} \gtrsim \ln \left(1+g_{\xi}^{-1}(x-y)\right)
$$

For the other direction we consider three cases:

(i) $g_{\xi}^{-1}(x-y) \leq 1$. Then we set $\zeta=\xi$ and

$$
f(\zeta) \approx \sqrt{g_{\xi}^{-1}(x-y)} \approx \ln \left(1+g_{\xi}^{-1}(x-y)\right)
$$

(ii) $g_{\xi}^{-1}(x-y)>1$ and $|x-y|<1$. Then we choose $\zeta$ so that $g_{\zeta}^{-1}(x-y)=1$. This gives

$$
\begin{aligned}
f(\zeta) & =1+\ln \frac{\langle\xi\rangle}{\langle\zeta\rangle} \leq 1+\ln \frac{g_{\xi}^{-1}(x-y)}{g_{\zeta}^{-1}(x-y)} \\
& =1+\ln g_{\xi}^{-1}(x-y) \approx \ln \left(1+g_{\xi}^{-1}(x-y)\right)
\end{aligned}
$$

(iii) $|x-y| \geq 1$. Then we take $\zeta=0$ which gives

$$
f(\zeta)=\ln \langle\xi\rangle+|x-y| \lesssim \ln \left(1+g_{\xi}^{-1}(x-y)\right)+|x-y|
$$

Pick now any path $\gamma$ which joins $(x, \xi)$ and $(y, \xi)$. Define

$$
\theta=\sup _{(z, \eta) \in \gamma} \angle(\xi, \eta), \quad r=\min _{(z, \eta) \in \gamma}\langle\eta\rangle
$$

We choose $\zeta \in[0, \xi]$ with

$$
\langle\zeta\rangle=\min \left\{r, \theta^{-2}\right\}
$$

Then the frequency projection of the trajectory $\gamma$ is contained above $\zeta$ in a sector centered at $\zeta$ and of angle $\langle\zeta\rangle^{-\frac{1}{2}}$. This implies that

$$
g_{\eta}^{-1} \gtrsim g_{\zeta}^{-1} \quad \forall(z, \eta) \in \gamma
$$


Taking advantage of the analysis in the proof of (1) it follows that

$$
l(\gamma) \gtrsim \sqrt{g_{\zeta}^{-1}(x-y)}+d(\xi, \zeta) \approx \sqrt{g_{\zeta}^{-1}(x-y)}+\ln \frac{\langle\xi\rangle}{\langle\zeta\rangle}=f(\zeta)
$$

which concludes the proof.

Later in the paper we want to use the distance $d$ to express phase space kernel bounds of pseudodifferential operators. For this purpose the last term in (2) proves inconvenient. Hence we introduce a second distance $\tilde{d}$ which is a technical modification of the distance $d$. The term $|x-y|$ arises when the geodesic moves between $x$ and $y$ at frequency close to 0 and speed 1 . We make this move less expensive by setting

$$
\begin{aligned}
\tilde{d}((x, \xi),(y, \eta))= & \min \left\{d((x, \xi),(y, \eta)), \inf _{x_{1}, y_{1}}\left\{d\left((x, \xi),\left(x_{1}, 0\right)\right)\right.\right. \\
& \left.\left.+\ln \left(1+\left|x_{1}-y_{1}\right|\right)+d\left(\left(y_{1}, 0\right),(y, \eta)\right)\right\}\right\}
\end{aligned}
$$

It is easy to prove that this is a distance since $d$ is a distance and $\ln (1+x)$ is subadditive in $\mathbb{R}^{+}$. By $(2)$ we have

$$
d\left((x, \xi),\left(x_{1}, 0\right)\right) \approx d(\xi, 0)+\left|x-x_{1}\right|
$$

therefore we can approximate $\tilde{d}$ with

$$
\begin{aligned}
\tilde{d}((x, \xi),(y, \eta))=\min \{ & d((x, \xi),(y, \eta)), \\
& d(\xi, 0)+\ln (1+|x-y|)+d(0, \eta)\}
\end{aligned}
$$

When $|x-y|<1$ the two distances are comparable. However, the long range behavior of $\tilde{d}$ is given by

$$
\tilde{d}((x, \xi),(y, \eta)) \approx d(\xi, \eta)+\ln \left(1+g_{\xi}^{-1}(x-y)\right)
$$

We also introduce an even version of $d$ and $\tilde{d}$,

$$
d_{\text {even }}(\xi, \eta)=\min \{d(\xi, \eta), d(\xi,-\eta)\}
$$

and

$$
\tilde{d}_{\text {even }}((x, \xi),(y, \eta))=\min \{\tilde{d}((x, \xi),(y, \eta)), \tilde{d}((x, \xi),(y,-\eta))\}
$$

Then we have

(6) $1+\tilde{d}_{\text {even }}((x, \xi),(y, \eta)) \approx \ln \left(g_{\xi}(\xi-\eta)+g_{\xi}^{-1}(x-y)+\frac{\langle\xi\rangle}{\langle\eta\rangle}+\frac{\langle\eta\rangle}{\langle\xi\rangle}\right)$

Lemma 2.6. For large enough $N$ we have

$$
\int_{\mathbb{R}^{2 n}} e^{-N \tilde{d}_{e v e n}((x, \xi),(y, \eta))} d y d \eta \lesssim 1
$$

and the similar bound for $\tilde{d}$. 
Proof. By (6) we have

$$
\int_{\mathbb{R}^{2 n}} e^{-N \tilde{d}_{\text {even }}((x, \xi),(y, \eta))} d y d \eta \lesssim \int_{\mathbb{R}^{2 n}}\left(1+g_{\xi}(\xi-\eta)+g_{\xi}^{-1}(x-y)\right)^{-c N} d y d \eta \lesssim 1
$$

\section{The PHASE SPACE TRANSFORM}

The coherent states adapted to the phase space structure induced by the metric $g$ are bump functions $\phi_{x, \xi}$ which are localized in frequency within $R_{\xi}$ and in position in $x+R_{\xi}^{-1}$. We certainly cannot have sharp localization at both ends. Unlike in the case of the classical Bargmann transform, we choose to have sharp frequency localization in order to prevent potentially troublesome concentrations at the origin.

Definition 3.1. Let $h$ be an $L^{2}$ normalized smooth function supported in $B\left(0, \frac{1}{16}\right)$. Then the coherent states $\phi_{x, \xi}$ are defined by

$$
\hat{\phi}_{x, \xi}(\eta)=\langle\eta\rangle^{-\frac{n+1}{4}} h\left(g_{\eta}(\xi-\eta)\right) e^{-i \eta x}
$$

We also introduce the notation

$$
\phi_{\xi}(\eta)=\langle\eta\rangle^{-\frac{n+1}{4}} h\left(g_{\eta}(\xi-\eta)\right)
$$

We note that $\phi_{x, \xi}(y)$ are not exactly $L^{2}$ normalized but

$$
\left\|\phi_{x, \xi}\right\|_{L^{2}} \approx 1
$$

One can also see that they can be represented in the form

$$
\phi_{x, \xi}(y)=\langle\xi\rangle^{\frac{n+1}{4}} k_{\xi}(y-x) e^{i \xi(y-x)}
$$

where $k_{\xi}$ is a smooth bump function on the $R_{\xi}^{-1}$ scale.

Lemma 2.1 allows us to describe the frequency support of the coherent states:

Lemma 3.2. For $\xi, \eta \in \mathbb{R}^{n}$ we have

$$
\hat{\phi}_{x, \xi}(\eta) \neq 0 \Longrightarrow \xi \in R_{\eta}, \eta \in R_{\xi}
$$

Now we can define our phase space transform:

Definition 3.3. The phase transform $T$ is

$$
T u(x, \xi)=\int_{\mathbb{R}^{n}} u(y) \overline{\phi_{x, \xi}(y)} d y, \quad \forall u \in \mathcal{S}\left(\mathbb{R}^{n}\right)
$$


We can also express $T u$ in terms of the Fourier transform of $u$,

$$
T u(x, \xi)=\int_{\mathbb{R}^{n}} \hat{u}(\eta) \overline{\hat{\phi}_{x, \xi}(\eta)} d \eta
$$

The adjoint operator $T^{*}$ is given by

$$
T^{*} f(y)=\int_{\mathbb{R}^{n}} f(x, \xi) \phi_{x, \xi}(y) d x d \xi
$$

It is easy to see that

Proposition 3.4. The following mapping properties hold:

$$
T: S\left(\mathbb{R}^{n}\right) \rightarrow S\left(\mathbb{R}^{2 n}\right), \quad T^{*}: S\left(\mathbb{R}^{2 n}\right) \rightarrow S\left(\mathbb{R}^{n}\right)
$$

By duality this allows us to extend $T$ and $T^{*}$ to linear operators

$$
T: S^{\prime}\left(\mathbb{R}^{n}\right) \rightarrow S^{\prime}\left(\mathbb{R}^{2 n}\right), \quad T^{*}: S^{\prime}\left(\mathbb{R}^{2 n}\right) \rightarrow S^{\prime}\left(\mathbb{R}^{n}\right)
$$

As for the classical Bargmann transform we have

Proposition 3.5. The phase space transform $T$ is an isometry

$$
T: L^{2}\left(\mathbb{R}^{n}\right) \longrightarrow L^{2}\left(\mathbb{R}^{2 n}\right) .
$$

Thus we have the inversion formula

$$
u(y)=\int_{\mathbb{R}^{2 n}} T u(x, \xi) \phi_{x, \xi}(y) d x d \xi
$$

Proof. A straightforward calculation using the Fourier inversion formula leads to:

$$
\begin{aligned}
\widehat{T^{*} T u} & (\zeta)=\int \hat{u}(\eta) \overline{\hat{\phi}_{x, \xi}(\eta)} \hat{\phi}_{x, \xi}(\zeta) d \eta d x d \xi \\
= & \int \hat{u}(\eta) e^{i x(\zeta-\eta)} h\left(g_{\zeta}(\xi-\zeta)\right) \overline{h\left(g_{\eta}(\xi-\eta)\right)}\langle\eta\rangle^{-\frac{n+1}{4}}\langle\zeta\rangle^{-\frac{n+1}{4}} d \eta d x d \xi \\
= & \langle\zeta\rangle^{-\frac{n+1}{2}} \hat{u}(\zeta) \int \mid h\left(\left.g_{\zeta}(\xi-\zeta)\right|^{2} d \xi\right. \\
= & \hat{u}(\zeta)
\end{aligned}
$$

\section{Symbol Classes AND PSEUdodifferential OPERATORS}

As a starting point we define the symbol class which is associated to the metric $g$. Precisely, we consider symbols which are smooth in $\xi$ on the $R_{\xi}$ scale and in $x$ on the $R_{\xi}^{-1}$ scale. To describe their size we need 
Definition 4.1. A function $m: \mathbb{R}^{2 n} \rightarrow \mathbb{R}^{+}$is slowly varying function with respect to the metric $g$ if

$$
d((x, \xi),(y, \eta)) \leq 1 \Rightarrow m(x, \xi) \approx m(y, \eta)
$$

and there exists $k$ so that

$$
m(x, 0) \lesssim m(y, 0)(1+|x-y|)^{k}
$$

Obvious examples of slowly varying functions are $1,\langle\xi\rangle^{s}$. Since within each unit ball $m$ can change by a fixed factor, we easily obtain

$$
m(x, \xi) \lesssim m(y, \eta) e^{C \tilde{d}((x, \xi),(y, \eta))}
$$

Definition 4.2. Let $m: \mathbb{R}^{2 n} \rightarrow \mathbb{R}^{+}$be a slowly varying function with respect to the metric $g$. The symbol $a \in C^{\infty}\left(\mathbb{R}^{2 n}\right)$ belongs to $S(m, g)$ if it satisfies the following estimates:

$$
\left.\mid \nabla^{\alpha} a(x, \xi)\right)\left.\right|_{g} \leq c_{\alpha} m(x, \xi)
$$

If $m=1$ then we simply write $S(g)$.

Unfortunately, the corresponding class of operators $O P S(g)$ is not so well behaved because a certain degree of concentration at frequency 0 is permitted for the corresponding pseudodifferential operator. This phenomena is similar to what happens in the case of $S_{1,1}^{0}$ symbols, only it gets worse here. To remedy this we consider the analogue of Hörmander's [8] $\tilde{S}_{1,1}^{0}$ class (see also [1], [4], [9]):

Definition 4.3. Let $m: \mathbb{R}^{2 n} \rightarrow \mathbb{R}^{+}$be an even slowly varying function with respect to the metric $g$. The symbol $a \in S(m, g)$ belongs to $\tilde{S}(m, g)$ if in addition it satisfies the following estimates:

$$
\left|S_{<\lambda}\left(D_{x}+\xi\right) a(x, \xi)\right| \leq c_{N} m(x, \xi)\left(\frac{\lambda}{\langle\xi\rangle}\right)^{N}, \quad 1 \leq \lambda \leq\langle\xi\rangle
$$

where $S_{<\lambda}$ is a smooth multiplier selecting the region $\langle\eta\rangle \lesssim \lambda$.

This is a condition which limits the part of $A(x, D)$ which takes high frequencies to low frequencies. It is dependent on the calculus that we use. The one above is adapted to the left calculus, but we would have to use a different one for the right calculus or say for the Weyl calculus. A useful example of symbols satisfying this extra condition is as follows:

Remark 4.4. Let $a \in S(m, g)$ so that $\hat{a}(\eta, \xi)$ is supported in $|\eta| \ll|\xi|$. Then $a \in \tilde{S}(m, g)$. This is because the expression in (13) vanishes if $\lambda \ll\langle\xi\rangle$. 
Our main result is to characterize the symbols in the $\tilde{S}(m, g)$ class in terms of the phase space transform.

Theorem 4.5. Let $A: S\left(\mathbb{R}^{n}\right) \rightarrow S^{\prime}\left(\mathbb{R}^{n}\right)$ be a linear operator. Let $m: \mathbb{R}^{2 n} \rightarrow \mathbb{R}^{+}$be an even slowly varying function with respect to the metric $g$. Then $A \in O P \tilde{S}(m, g)$ if and only if the kernel $K$ of $T A T^{*}$ satisfies the following bounds:

$$
\left|K\left(x_{1}, \xi_{1}, x_{2}, \xi_{2}\right)\right| \leq c_{N} m\left(x_{1}, \xi_{1}\right) e^{-N \tilde{d}_{e v e n}\left(\left(x_{1}, \xi_{1}\right),\left(x_{2}, \xi_{2}\right)\right)}
$$

for all $N \in \mathbb{N}$.

We note that by $(11)$ the factors $m\left(x_{1}, \xi_{1}\right)$ and $m\left(x_{2}, \xi_{2}\right)$ are interchangeable. We use the even distance here because for such operators the input from frequency $\xi$ to frequency $-\xi$ is nontrivial. This is a reflection of the fact that $R_{-\xi}$ is contained in a dilation of $R_{\xi}$. Using the expression for $\tilde{d}_{\text {even }}$ we also rewrite the bound (14) in the form

$$
\left|K\left(x_{1}, \xi_{1}, x_{2}, \xi_{2}\right)\right| \leq c_{N} \frac{m\left(x_{1}, \xi_{1}\right) \min \left\{\left\langle\xi_{1}\right\rangle /\left\langle\xi_{2}\right\rangle,\left\langle\xi_{2}\right\rangle /\left\langle\xi_{1}\right\rangle\right\}^{N}}{\left(1+g_{\xi_{2}}\left(\xi_{1}-\xi_{2}\right)+g_{\xi_{1}}^{-1}\left(x_{1}-x_{2}\right)\right)^{N}}
$$

The above theorem immediately leads to

Corollary 4.6. Let $A: S\left(\mathbb{R}^{n}\right) \rightarrow S^{\prime}\left(\mathbb{R}^{n}\right)$ be a linear operator. Then $A \in O P \tilde{S}(m, g)$ iff $A^{*} \in O P \tilde{S}(m, g)$.

As far as multiplicative properties are concerned, we note that while

$$
S\left(m_{1}, g\right) \cdot S\left(m_{2}, g\right) \subset S\left(m_{1} m_{2}, g\right)
$$

the similar property for operators can fail. In the terminology of Hörmander [7], section 18.5, this is connected to the fact that our metric on the cotangent space $T^{*} \mathbb{R}^{n}$ is not temperate with respect to the symplectic form.

The opposite happens with the $\tilde{S}$ symbol classes. While

$$
\tilde{S}\left(m_{1}, g\right) \cdot \tilde{S}\left(m_{2}, g\right) \not \subset \tilde{S}\left(m_{1} m_{2}, g\right)
$$

we do have the more interesting property:

Corollary 4.7. Let $m_{1}, m_{2}$ be even slowly varying functions with respect to the metric $g$. Then

$$
O P \tilde{S}\left(m_{1}, g\right) O P \tilde{S}\left(m_{2}, g\right) \subset O P \tilde{S}\left(m_{1} m_{2}, g\right) .
$$

Proof. For $i=1,2$ we consider symbols $a_{i} \in \tilde{S}\left(m_{i}, g\right)$ and denote by $K_{i}$ the kernels of $T A_{i}(x, D) T^{*}$. Then the kernel $K$ of the operator $T A_{1}(x, D) A_{2}(x, D) T^{*}$ is given by

$$
K\left(x_{1}, \xi_{1}, x_{2}, \xi_{2}\right)=\int K_{1}\left(x_{1}, \xi_{1}, x, \xi\right) K_{2}\left(x, \xi, x_{2}, \xi_{2}\right) d x d \xi
$$


We take absolute values and use (14) for $K_{1}$ and $K_{2}$. Taking advantage of the triangle inequality for $\tilde{d}_{\text {even }}$ we obtain

$$
\begin{aligned}
& \left|K\left(x_{1}, \xi_{1}, x_{2}, \xi_{2}\right)\right| \\
\lesssim & c_{N} m_{1}\left(x_{1}, \xi_{1}\right) m_{2}\left(x_{2}, \xi_{2}\right) \int e^{-N \tilde{d}_{e v e n}\left(\left(x_{1}, \xi_{1}\right),(x, \xi)\right)} e^{-2 N \tilde{d}_{\text {even }}\left((x, \xi),\left(x_{2}, \xi_{2}\right)\right)} d x d \xi \\
\lesssim & c_{N} m_{1}\left(x_{1}, \xi_{1}\right) m_{2}\left(x_{2}, \xi_{2}\right) e^{-N \tilde{d}_{\text {even }}\left(\left(x_{1}, \xi_{1}\right),\left(x_{2}, \xi_{2}\right)\right)} \int e^{-N \tilde{d}_{\text {even }}\left((x, \xi),\left(x_{2}, \xi_{2}\right)\right)} d x d \xi
\end{aligned}
$$

By lemma 2.6 the last integral has size $O(1)$. Also $m_{1}$ and $m_{2}$ are slowly varying so their arguments are interchangeable. Hence the proof is concluded.

Next we turn our attention to Sobolev space estimates. The simplest one is:

Theorem 4.8. Let $a \in \tilde{S}(g)$. Then

$$
A(x, D): L^{2} \rightarrow L^{2}
$$

Proof. We write $A(x, D)=T^{*}\left(T A(x, D) T^{*}\right) T$. Since $T$ is an isometry, $A(x, D)$ is $L^{2}$ bounded if and only if $T A(x, D) T^{*}$ is $L^{2}$ bounded. This follows from the kernel bounds

$$
\begin{aligned}
& \sup _{x_{1}, \xi_{1}} \int\left|K\left(x_{1}, \xi_{1}, x_{2}, \xi_{2}\right)\right| d x_{2} d \xi_{2}<\infty \\
& \sup _{x_{2}, \xi_{2}} \int\left|K\left(x_{1}, \xi_{1}, x_{2}, \xi_{2}\right)\right| d x_{1} d \xi_{1}<\infty
\end{aligned}
$$

These are an immediate consequence of the kernel bound (14) and of Lemma 2.6.

Since we trivially have

$$
\langle\xi\rangle^{s} \in S\left(\langle\xi\rangle^{s}, g\right), \quad s \in \mathbb{R}
$$

we can use the composition theorem to conclude that

Corollary 4.9. Let $a \in \tilde{S}(g)$. Then

$$
A(x, D): H^{s} \rightarrow H^{s}, \quad s \in \mathbb{R}
$$

We turn our attention for a moment to the $S(g)$ symbol class. As a byproduct of the proof of Theorem 4.5 one obtains

Corollary 4.10. If $a \in S(g)$ then the kernel $K$ of $T A T^{*}$ satisfies

$$
\left|K\left(x_{1}, \xi_{1}, x_{2}, \xi_{2}\right)\right| \leq c_{N} \frac{\left(\left\langle\xi_{2}\right\rangle /\left\langle\xi_{1}\right\rangle\right)^{\frac{n+1}{4}}}{\left(1+g_{\xi_{2}}\left(\xi_{1}-\xi_{2}\right)+g_{\xi_{1}}^{-1}\left(x_{1}-x_{2}\right)\right)^{N}}
$$


Since

$$
\frac{1}{1+g_{\xi_{2}}\left(\xi_{1}-\xi_{2}\right)} \lesssim \frac{\left\langle\xi_{2}\right\rangle^{2}}{\left\langle\xi_{1}\right\rangle^{2}}
$$

the bound (16) is as strong as (14) in the region $\left\{\left|\xi_{2}\right| \lesssim\left|\xi_{1}\right|\right\}$. This leads to the following alternative characterization of $\tilde{S}_{g}$ :

Theorem 4.11. Let $A: S \rightarrow S^{\prime}$ be a linear operator. Then $A \in$ $O P \tilde{S}(g)$ iff $A \in O P S(g)$ and $A^{*} \in O P S(g)$.

Remark 4.12. If $a \in S(g)$ then one can prove that

$$
a(x, D): H^{s}\left(\mathbb{R}^{n}\right) \longrightarrow H^{s}\left(\mathbb{R}^{n}\right), \quad(\forall) s>\frac{n-2}{2}
$$

A direct argument using (16) only yields the $\frac{n-1}{2}$ threshold, and a slightly finer analysis is needed for the optimal result.

While the even phase space structure is compatible with the calculus of pseudodifferential operators, things change if we want to consider Fourier integral operators. Indeed, there is no reason to restrict ourselves to canonical transformations which commute with the symmetry with respect to the origin. Hence we introduce a third and smaller class of pseudodifferential operators:

Definition 4.13. Let $m: \mathbb{R}^{2 n} \rightarrow \mathbb{R}^{+}$be an even slowly varying function with respect to the metric $g$. The symbol $a \in \tilde{S}(m, g)$ belongs to $\tilde{\tilde{S}}(m, g)$ if in addition it satisfies the following estimates:

$$
\left|S_{<\epsilon\langle\xi\rangle}\left(D_{x}+(1+\epsilon) \xi\right) a(x, \xi)\right| \leq c_{N} m(x, \xi)\langle\xi\rangle^{-N}, \quad\langle\xi\rangle^{-\frac{1}{2}}<\epsilon<\langle\xi\rangle^{\frac{1}{2}}
$$

Then we have the characterization theorem

Theorem 4.14. Let $A: S\left(\mathbb{R}^{n}\right) \rightarrow S^{\prime}\left(\mathbb{R}^{n}\right)$ be a linear operator. Let $m: \mathbb{R}^{2 n} \rightarrow \mathbb{R}^{+}$be a slowly varying function with respect to the metric $g$. Then $A \in O P \tilde{\tilde{S}}(m, g)$ if and only if the kernel $K$ of $T A T^{*}$ satisfies the following bounds:

$$
\left|K\left(x_{1}, \xi_{1}, x_{2}, \xi_{2}\right)\right| \leq c_{N} m\left(x_{1}, \xi_{1}\right) e^{-N \tilde{d}\left(\left(x_{1}, \xi_{1}\right),\left(x_{2}, \xi_{2}\right)\right)}
$$

for all $N \in \mathbb{N}$.

The proof essentially repeats the proof of Theorem 4.5 and is omitted.

We now return to the proof of our main result. 
Proof of Theorem 4.5. a) Consider a symbol $a \in S(m, g)$. The kernel $K$ of $T A(x, D) T^{*}$ has the form

$$
K\left(x_{1}, \xi_{1}, x_{2}, \xi_{2}\right)=\int e^{i x \cdot(\xi-\eta)} a(x, \xi) \overline{\hat{\phi}}_{x_{1}, \xi_{1}}(\eta) \hat{\phi}_{x_{2}, \xi_{2}}(\xi) d x d \xi d \eta
$$

Given our choice of the coherent states, we can rewrite it in the form

$$
K=\left[\left|R_{\xi_{1}}\right|\left|R_{\xi_{2}}\right|\right]^{-\frac{1}{2}} \int e^{i\left[\xi\left(x-x_{2}\right)+\eta\left(x_{1}-x\right)\right]} a(x, \xi) G_{\xi_{1}, \xi_{2}}(\eta, \xi) d x d \xi d \eta
$$

where

$$
G_{\xi_{1}, \xi_{2}}(\xi, \eta)=\left(\frac{\left\langle\xi_{1}\right\rangle\left\langle\xi_{2}\right\rangle}{\langle\eta\rangle\langle\xi\rangle}\right)^{\frac{n+1}{4}} h\left(g_{\xi}\left(\xi_{2}-\xi\right)\right) \overline{h\left(g_{\eta}\left(\xi_{1}-\eta\right)\right)}
$$

We note that $G_{\xi_{1}, \xi_{2}}$ is a size one bump function with support in $R_{\xi_{1}} \times R_{\xi_{2}}$ and smooth on the same scale. This is all that we need in the sequel. The fact that $a$ depends also on $\xi$ makes no difference, since $a(x, \xi)$ is also smooth in $R_{\xi_{2}}$ on the $R_{\xi_{2}}$ scale and has size $m\left(x, \xi_{2}\right)$.

a1) An uniform bound on $K$. The Fourier transform of a bump function is a bump function on the dual scale. Hence, taking the Fourier transform with respect to $\xi$ and $\eta$ in $K$ we obtain

$$
|K| \lesssim\left[\left|R_{\xi_{1}}\right|\left|R_{\xi_{2}}\right|\right]^{\frac{1}{2}} m\left(x_{2}, \xi_{2}\right) \int\left(1+g_{\xi_{1}}^{-1}\left(x_{1}-x\right)\right)^{-N}\left(1+g_{\xi_{2}}^{-1}\left(x_{2}-x\right)\right)^{-N} d x
$$

Estimating each of the two bump functions under the integral in $L^{2}$ yields

$$
|K| \lesssim\left[\left|R_{\xi_{1}}\right|\left|R_{\xi_{2}}\right|\right]^{\frac{1}{2}} m\left(x_{2}, \xi_{2}\right)\left[\left|R_{\xi_{1}}\right|\left|R_{\xi_{2}}\right|\right]^{-\frac{1}{2}} \lesssim m\left(x_{2}, \xi_{2}\right)
$$

Estimating the first bump in $L^{\infty}$ and the second in $L^{1}$ yields the better bound

$$
|K| \lesssim m\left(x_{2}, \xi_{2}\right)\left|R_{\xi_{1}}\right|^{\frac{1}{2}}\left|R_{\xi_{2}}\right|^{-\frac{1}{2}}
$$

a2) Frequency decay for $K$. We begin with the relation

$$
\left(1-\Delta_{g_{\xi_{2}}^{-1}}\right)^{N} e^{i x(\xi-\eta)}=\left(1+g_{\xi_{2}}(\eta-\xi)\right)^{N} e^{i x(\xi-\eta)}
$$

Substituting this in $K$ and integrating by parts yields

$$
\begin{array}{r}
K=\left[\left|R_{\xi_{1}}\right|\left|R_{\xi_{2}}\right|\right]^{-\frac{1}{2}} \int e^{i\left[\xi\left(x-x_{2}\right)+\eta\left(x_{1}-x\right)\right]}\left(1-\Delta_{g_{\xi_{2}}^{-1}}\right)^{N} a(x, \xi) \\
\left(1+g_{\xi_{2}}(\eta-\xi)\right)^{-N} G_{\xi_{1}, \xi_{2}}(\eta, \xi) d x d \xi d \eta
\end{array}
$$

For $\xi \in R_{\xi_{2}}$ the symbol $\left(1-\Delta_{g_{\xi_{2}}^{-1}}\right)^{N} a(x, \xi)$ has the same bounds and regularity as $a(x, \xi)$. Also, by Lemma 2.4 , within $R_{\xi_{1}} \times R_{\xi_{2}}$ the function $\left(1+g_{\xi_{2}}(\eta-\xi)\right)^{-N}$ is smooth and has size $\left(1+g_{\xi_{2}}\left(\xi_{1}-\xi_{2}\right)\right)^{-N}$. Then 
we can include it in $G$ and argue as in case (a1) to obtain the analogue of $(20)$,

$$
|K| \lesssim\left|R_{\xi_{1}}\right|^{\frac{1}{2}}\left|R_{\xi_{2}}\right|^{-\frac{1}{2}} m\left(x_{2}, \xi_{2}\right)\left(1+g_{\xi_{2}}\left(\xi_{1}-\xi_{2}\right)\right)^{-N}
$$

a3) Spatial decay for $K$. Here we return to the bound (19) and improve the estimate for the integral. For this we use the bound in Lemma 2.3. This yields

$$
\left(1+g_{\xi_{1}}^{-1}\left(x_{2}-x\right)\right) \lesssim\left(1+g_{\xi_{2}}^{-1}\left(x_{2}-x\right)\right)\left(1+g_{\xi_{2}}\left(\xi_{1}-\xi_{2}\right)\right)
$$

and by the triangle inequality

$$
\left(1+g_{\xi_{1}}^{-1}\left(x_{1}-x_{2}\right)\right) \lesssim\left(1+g_{\xi_{2}}^{-1}\left(x_{2}-x\right)\right)\left(1+g_{\xi_{2}}\left(\xi_{1}-\xi_{2}\right)\right)\left(1+g_{\xi_{1}}^{-1}\left(x_{1}-x\right)\right)
$$

By (19) this implies that

$$
|K| \lesssim\left[\left|R_{\xi_{1}}\right|\left|R_{\xi_{2}}\right|\right]^{\frac{1}{2}} \int \frac{m\left(x_{2}, \xi_{2}\right)\left(1+g_{\xi_{2}}\left(\xi_{1}-\xi_{2}\right)\right)^{N}}{\left(1+g_{\xi_{1}}^{-1}\left(x_{1}-x_{2}\right)\right)^{N}\left(1+g_{\xi_{2}}^{-1}\left(x_{2}-x\right)\right)^{N}} d x
$$

After integration in $x$ we get

$$
|K| \lesssim m\left(x_{2}, \xi_{2}\right)\left|R_{\xi_{1}}\right|^{\frac{1}{2}}\left|R_{\xi_{2}}\right|^{-\frac{1}{2}}\left(1+g_{\xi_{2}}\left(\xi_{1}-\xi_{2}\right)\right)^{N}\left(1+g_{\xi_{1}}^{-1}\left(x_{1}-x_{2}\right)\right)^{-N}
$$

We can combine (22) and (23) to obtain

$$
|K| \lesssim m\left(x_{2}, \xi_{2}\right)\left|R_{\xi_{1}}\right|^{\frac{1}{2}}\left|R_{\xi_{2}}\right|^{-\frac{1}{2}}\left(1+g_{\xi_{2}}\left(\xi_{1}-\xi_{2}\right)\right)^{-N}\left(1+g_{\xi_{1}}^{-1}\left(x_{1}-x_{2}\right)\right)^{-N}
$$

which is the optimal bound under the assumption $a \in S(m, g)$ (see Corollary 4.10).

Since

$$
1+g_{\xi_{2}}\left(\xi_{1}-\xi_{2}\right) \geq \frac{1+\xi_{1}^{2}}{1+\xi_{2}^{2}}
$$

this bound gives rapid decay for $K$ as $\left|\xi_{2}\right| /\left|\xi_{1}\right| \rightarrow 0$. To conclude the proof of the bound for $K$ it remains to obtain the rapid decay as $\left|\xi_{2}\right| /\left|\xi_{1}\right| \rightarrow \infty$. This is the only place in the argument where we use the $\tilde{S}(m, g)$ structure.

a4) High-low frequency bounds. Suppose that $\left\langle\xi_{1}\right\rangle \ll\left\langle\xi_{2}\right\rangle$. Choose $\lambda=4\left\langle\xi_{1}\right\rangle$. Then

$$
\hat{\phi}_{x_{1}, \xi_{1}}(\eta)=S_{<\lambda}(\eta) \hat{\phi}_{x_{1}, \xi_{1}}(\eta)
$$

Substituting this in the expression for $K, S_{<\lambda}$ can be interpreted as a multiplier in the $x$ variable. Then

$$
K\left(x_{1}, \xi_{1}, x_{2}, \xi_{2}\right)=\int e^{-i x \eta}\left(S_{<\lambda} e^{i x \xi} a(x, \xi)\right) \overline{\hat{\phi}_{x_{1}, \xi_{1}}(\eta)} \hat{\phi}_{x_{2}, \xi_{2}}(\xi) d x d \xi d \eta
$$

Thus we obtain a similar expression but with $a(x, \xi)$ replaced by

$$
e^{-i x \xi} S_{<\lambda}\left(e^{i x \xi} a(x, \xi)\right)=S_{<\lambda}\left(D_{x}+\xi\right) a(x, \xi)
$$


Then we can simply apply the bound (23) provided we know the stronger symbol estimate

$$
\left|\nabla^{k} S_{<\lambda}\left(D_{x}+\xi\right) a(x, \xi)\right|_{g} \lesssim c_{k, N} m(x, \xi)(\lambda /\langle\xi\rangle)^{N}
$$

For $k=0$ this is exactly our hypothesis (13). For $k>0$ we begin with (12) which yields

$$
\left|S_{<\lambda}\left(D_{x}+\xi\right) \nabla^{k} a(x, \xi)\right|_{g} \lesssim c_{N} m(x, \xi)(\lambda /\langle\xi\rangle)^{-k_{0}}
$$

for some large $k$. Here the large factor on the right measures the possible change in $m(x, \xi)$ within a $\lambda^{-1}$ ball, in which the kernel of the multiplier is concentrated.

The $x$ derivatives commute with the multiplier. The $\xi$ derivatives yield extra $\lambda^{-1}$ factors, which combined with the additional $\langle\xi\rangle$ factor coming from $g$ yield

$$
\left|\nabla^{k} S_{<\lambda}\left(D_{x}+\xi\right) a(x, \xi)\right|_{g} \lesssim c_{k, N} m(x, \xi)(\lambda /\langle\xi\rangle)^{-2 k-k_{0}}
$$

Interpolating this with (13) for $k=0$ on unit $g$-balls yields (25) and concludes the proof of the bounds for $K$.

b) Assume now that $K$ verifies (14). We interpret $A$ as a pseudodifferential operator with symbol $a(x, \xi)$ and prove that $a$ satisfies (12) and (13). For $a$ we have the following representation:

$$
a(x, \xi)=\int e^{-i x \cdot(\xi-\eta)} K\left(x_{1}, \xi_{1}, x_{2}, \xi_{2}\right) \hat{\phi}_{x_{1}, \xi_{1}}(\eta) \overline{\hat{\phi}_{x_{2}, \xi_{2}}(\xi)} d x_{i} d \xi_{i} d \eta
$$

With the same notations as in the case of $K$ this gives

$$
\begin{aligned}
& a(x, \xi)=\int\left[\left|R_{\xi_{1}}\right|\left|R_{\xi_{2}}\right|\right]^{-\frac{1}{2}} e^{-i\left[\xi\left(x-x_{2}\right)+\eta\left(x_{1}-x\right)\right]} K\left(x_{1}, \xi_{1}, x_{2}, \xi_{2}\right) \\
& \overline{G_{\xi_{1}, \xi_{2}}(\eta, \xi)} d x_{i} d \xi_{i} d \eta
\end{aligned}
$$

Since we only have pointwise bounds on $K$, the sole nontrivial oscillatory integral here is the one with respect to $\eta$.

b1) An uniform bound on a. Taking the Fourier transform with respect to $\eta$ yields

$$
|a(x, \xi)| \lesssim \int \frac{1_{R_{\xi_{2}}}(\xi)\left|R_{\xi_{1}}\right|^{\frac{1}{2}}\left|R_{\xi_{2}}\right|^{-\frac{1}{2}}\left|K\left(x_{1}, \xi_{1}, x_{2}, \xi_{2}\right)\right|}{\left(1+g_{\xi_{1}}^{-1}\left(x-x_{1}\right)\right)^{N}} d x_{i} d \xi_{i}
$$

We replace $K$ with its bound to obtain

$$
\begin{gathered}
|a(x, \xi)| \lesssim \int \frac{1_{R_{\xi_{2}}}(\xi)\left|R_{\xi_{1}}\right| \frac{1}{2}\left|R_{\xi_{2}}\right|^{-\frac{1}{2}} \min \left\{\left\langle\xi_{1}\right\rangle /\left\langle\xi_{2}\right\rangle,\left\langle\xi_{2}\right\rangle /\left\langle\xi_{1}\right\rangle\right\}^{N} m\left(x_{2}, \xi_{2}\right)}{\left(1+g_{\xi_{2}}\left(\xi_{2}-\xi_{1}\right)\right)^{N}} \\
\frac{1}{\left(1+g_{\xi_{1}}^{-1}\left(x_{1}-x_{2}\right)\right)^{N}\left(1+g_{\xi_{1}}^{-1}\left(x-x_{1}\right)\right)^{N}} d x_{i} d \xi_{i}
\end{gathered}
$$


where due to the "min" factor we are allowed to freely interchange $g_{\xi_{1}}$ and $g_{\xi_{2}}$. We integrate successively with respect to $x_{2}$ and then $x_{1}$ to obtain

$$
|a(x, \xi)| \lesssim \int \frac{1_{R_{\xi_{2}}}(\xi) \min \left\{\left\langle\xi_{1}\right\rangle /\left\langle\xi_{2}\right\rangle,\left\langle\xi_{2}\right\rangle /\left\langle\xi_{1}\right\rangle\right\}^{N} m\left(x, \xi_{2}\right)}{\left|R_{\xi_{1}}\right|^{\frac{3}{2}}\left|R_{\xi_{2}}\right|^{\frac{1}{2}}\left(1+g_{\xi_{2}}\left(\xi_{2}-\xi_{1}\right)\right)^{N}} d \xi_{i}
$$

Since

$$
\left|R_{\xi_{1}}\right|^{-\frac{3}{2}}\left|R_{\xi_{2}}\right|^{-\frac{1}{2}} \min \left\{\left\langle\xi_{1}\right\rangle /\left\langle\xi_{2}\right\rangle,\left\langle\xi_{2}\right\rangle /\left\langle\xi_{1}\right\rangle\right\}^{N} \lesssim\left|R_{\xi_{2}}\right|^{-2}
$$

we also integrate in $\xi_{1}$ to obtain

$$
|a(x, \xi)| \lesssim m(x, \xi) \int 1_{R_{\xi_{2}}}(\xi)\left|R_{\xi_{2}}\right|^{-1} d \xi_{2} \lesssim m(x, \xi)
$$

b2) The $x$ derivatives of $a$. Using the relation (21) we can write

$$
\begin{gathered}
\left(1-\Delta_{g_{\xi}^{-1}}\right)^{k} a(x, \xi)=\int e^{-i x \cdot(\xi-\eta)} K\left(x_{1}, \xi_{1}, x_{2}, \xi_{2}\right)\left(1+g_{\xi}(\xi-\eta)\right)^{k} \\
\hat{\phi}_{x_{1}, \xi_{1}}(\eta) \overline{\hat{\phi}_{x_{2}, \xi_{2}}(\xi)} d x_{i} d \xi_{i} d \eta
\end{gathered}
$$

By Lemma 2.4 the factor $\left(1+g_{\xi}(\xi-\eta)\right)^{k}$ is smooth in $\eta \in R_{\xi_{1}}$ on the $R_{\xi_{1}}$ scale so we can introduce it in $G$. Its size is comparable to

$$
\left(1+g_{\xi}(\xi-\eta)\right) \approx\left(1+g_{\xi_{2}}\left(\xi_{2}-\xi_{1}\right)\right) \quad \xi \in R_{\xi_{2}}, \eta \in R_{\xi_{1}}
$$

Hence it yields an additional factor of

$$
\left(1+g_{\xi_{2}}\left(\xi_{2}-\xi_{1}\right)\right)^{k}
$$

We continue the estimate as in (b1). The above additional factor is controlled by the off diagonal decay of $K$. Hence we obtain the same bound,

$$
\left|\left(1-\Delta_{g_{\xi}^{-1}}\right)^{k} a(x, \xi)\right| \lesssim m(x, \xi)
$$

b3) The $\xi$ derivatives of a.

$$
\begin{aligned}
\left(1-\Delta_{g_{\xi}}\right)^{k} a(x, \xi)= & \int e^{i x \eta} K\left(x_{1}, \xi_{1}, x_{2}, \xi_{2}\right) \hat{\phi}_{x_{1}, \xi_{1}}(\eta) \\
& \left(1-\Delta_{g_{\xi}}\right)^{k}\left(e^{-i x \xi} \overline{\hat{\phi}_{x_{2}, \xi_{2}}(\xi)}\right) d x_{i} d \xi_{i} d \eta
\end{aligned}
$$

We evaluate the differentiated term. Since

$$
e^{-i x \xi} \overline{\hat{\phi}_{x_{2}, \xi_{2}}(\xi)}=e^{i\left(x_{2}-x\right) \xi} \overline{h\left(g_{\xi}\left(\xi-\xi_{2}\right)\right)}
$$

and $g_{\xi}\left(\xi-\xi_{2}\right)$ is smooth in $\xi$ on the $R_{\xi}$ scale, we obtain

$$
\left|\left(1-\Delta_{g_{\xi}}\right)^{k}\left(e^{-i x \xi} \hat{\phi}_{x_{2}, \xi_{2}}(\xi)\right)\right| \lesssim\left(1+g_{\xi}^{-1}\left(x_{2}-x\right)\right)^{k} 1_{R_{\xi}}\left(\xi_{2}\right)
$$


We use this and continue as in (b1). The additional factor

$$
\left(1+g_{\xi}^{-1}\left(x_{2}-x\right)\right)^{k}
$$

is again negligible due to the off diagonal decay of $K$, so we obtain

$$
\left|\left(1-\Delta_{g_{\xi}}\right)^{k} a(x, \xi)\right| \lesssim m(x, \xi)
$$

b4) Additional decay near frequency $\xi$. From (26) we obtain

$$
\begin{aligned}
S_{<\lambda}\left(D_{x}+\xi\right) a(x, \xi)= & \int e^{-i x \cdot(\xi-\eta)} K\left(x_{1}, \xi_{1}, x_{2}, \xi_{2}\right) \\
& S_{<\lambda}(\eta) \hat{\phi}_{x_{1}, \xi_{1}}(\eta) \overline{\hat{\phi}_{x_{2}, \xi_{2}}(\xi)} d x_{i} d \xi_{i} d \eta \\
= & \int e^{-i x \cdot(\xi-\eta)} S_{<4 \lambda}\left(\xi_{1}\right) K\left(x_{1}, \xi_{1}, x_{2}, \xi_{2}\right) \\
& S_{<\lambda}(\eta) \hat{\phi}_{x_{1}, \xi_{1}}(\eta) \overline{\hat{\phi}_{x_{2}, \xi_{2}}(\xi)} d x_{i} d \xi_{i} d \eta
\end{aligned}
$$

We can include $S_{<\lambda}(\eta)$ in $\overline{\hat{\phi}_{x_{1}, \xi_{1}}(\eta)}$. Since $\xi_{1}$ is restricted to $\left\langle\xi_{1}\right\rangle \leq \lambda$, we can repeat the estimates in (b1) with the additional factor

$$
\left(\frac{\lambda}{\left\langle\xi_{1}\right\rangle}\right)^{N}
$$

coming from the "min" factor in the bound for $K$.

This concludes the proof of the theorem.

\section{Fourier INTEGRAL OPERATORS}

A canonical transformation of the phase space is a map which preserves the symplectic form

$$
\sigma=d x \wedge d \xi
$$

Here we introduce canonical transformations which are adapted to the metric $g$.

Definition 5.1. A locally Lipschitz canonical transformation

$$
\chi: T^{*} \mathbb{R}^{n} \rightarrow T^{*} \mathbb{R}^{n}
$$

is called $g$-Lipschitz if the following conditions are satisfied:

(i) If $(y, \eta)=\chi(x, \xi)$ then $\langle\eta\rangle \approx\langle\xi\rangle$.

(ii) If in addition $\left(y_{1}, \eta_{1}\right)=\chi\left(x_{1}, \xi_{1}\right)$ then

$$
d\left((y, \eta),\left(y_{1}, \eta_{1}\right)\right) \lesssim d\left((x, \xi),\left(x_{1}, \xi_{1}\right)\right)
$$

If $\chi$ is a diffeomorphism and both $\chi$ and $\chi^{-1}$ are $g$-Lipschitz then we say that $\chi$ is $g$-bi-Lipschitz. A simple but useful observation is 
Lemma 5.2. If $\chi$ is g-Lipschitz then it is also $\tilde{d}$-Lipschitz.

Proof. We need to show that

$$
\tilde{d}\left(\chi\left(x_{1}, \xi_{1}\right), \chi\left(x_{2}, \xi_{2}\right)\right) \lesssim \tilde{d}\left(\left(x_{1}, \xi_{1}\right),\left(x_{2}, \xi_{2}\right)\right)
$$

If

$$
\tilde{d}\left(\left(x_{1}, \xi_{1}\right),\left(x_{2}, \xi_{2}\right)\right) \gtrsim\left|x_{1}-x_{2}\right|
$$

then

$$
\tilde{d}\left(\left(x_{1}, \xi_{1}\right),\left(x_{2}, \xi_{2}\right)\right) \approx d\left(\left(x_{1}, \xi_{1}\right),\left(x_{2}, \xi_{2}\right)\right)
$$

and the proof is finished.

Otherwise, we must have $\left|x_{1}-x_{2}\right| \gg 1$ and, by (4),

$$
\begin{aligned}
\tilde{d}\left(\left(x_{1}, \xi_{1}\right),\right. & \left.\left(x_{2}, \xi_{2}\right)\right) \approx d\left(\left(x_{1}, \xi_{1}\right),\left(x_{1}, 0\right)\right)+d\left(\left(x_{2}, \xi_{2}\right),\left(x_{2}, 0\right)\right)+\ln \left|x_{1}-x_{2}\right| \\
& \gtrsim d\left(\chi\left(x_{1}, \xi_{1}\right), \chi\left(x_{1}, 0\right)\right)+d\left(\chi\left(x_{2}, \xi_{2}\right), \chi\left(x_{2}, 0\right)\right)+\ln \left|x_{1}-x_{2}\right| \\
& \gtrsim \tilde{d}\left(\chi\left(x_{1}, \xi_{1}\right), \chi\left(x_{1}, 0\right)\right)+\tilde{d}\left(\chi\left(x_{2}, \xi_{2}\right), \chi\left(x_{2}, 0\right)\right)+\ln \left|x_{1}-x_{2}\right|
\end{aligned}
$$

Hence we are left with proving that

$$
\tilde{d}\left(\chi\left(x_{1}, 0\right), \chi\left(x_{2}, 0\right)\right) \lesssim \ln \left|x_{1}-x_{2}\right|
$$

But by property (i) above we can neglect the frequency components on the left and write

$$
\begin{aligned}
\tilde{d}\left(\chi\left(x_{1}, 0\right), \chi\left(x_{2}, 0\right)\right) & \approx \ln d\left(\chi\left(x_{1}, 0\right), \chi\left(x_{2}, 0\right)\right)+O(1) \\
& \lesssim \ln d\left(\left(x_{1}, 0\right),\left(x_{2}, 0\right)\right)+O(1) \\
& \lesssim \ln \left|x_{1}-x_{2}\right|
\end{aligned}
$$

This concludes the proof.

To such canonical transformations we associate classes of Fourier integral operators.

Definition 5.3. Let $\chi$ be a g-Lipschitz canonical transformation and $m: T^{*} \mathbb{R}^{n} \rightarrow \mathbb{R}^{+}$be slowly varying. Let $A: S\left(\mathbb{R}^{n}\right) \rightarrow S^{\prime}\left(\mathbb{R}^{n}\right)$ be a linear operator. We say that $A \in F I O(m, g, \chi)$ if the kernel $K$ of $T A T^{*}$ satisfies

$$
|K(y, \eta, x, \xi)| \leq c_{N} m(x, \xi) e^{-N \tilde{d}((y, \eta), \chi(x, \xi)))}, \quad N \in \mathbb{N}
$$

We note that in the case when $\chi$ is the identity we have

$$
F I O(m, g, I)=O P \tilde{\tilde{S}}(m, g)
$$

This definition allows us to quickly establish algebra properties of Fourier integral operators. 
Theorem 5.4. Let $A_{i} \in F I O\left(m_{i}, g, \chi_{i}\right)$ for $i=1,2$. Then

$$
A_{1} A_{2} \in F I O(m, g, \chi), \quad m=\left(m_{1} \circ \chi_{2}\right) m_{2}, \quad \chi=\chi_{1} \circ \chi_{2} .
$$

Proof. Let $K_{i}$ be the kernels of $T A_{i} T^{*}$ for $i=1,2$. Then the kernel $K$ of $T A_{1} A_{2} T^{*}$ is given by

$$
K(y, \eta, x, \xi)=\int K_{1}(y, \eta, z, \zeta) K_{2}(z, \zeta, x, \xi) d z d \zeta
$$

We use the bounds in Definition 5.3 for $K_{1}$ and $K_{2}$. This gives $|K(y, \eta, x, \xi)| \lesssim \int m_{1}(z, \zeta) m_{2}(x, \xi) e^{-N \tilde{d}\left((y, \eta), \chi_{1}(z, \zeta)\right)} e^{-N \tilde{d}\left((z, \zeta), \chi_{2}(x, \xi)\right)} d z d \zeta$

From the triangle inequality and the Lipschitz property of $\chi_{1}$ we obtain

$$
\tilde{d}\left((y, \eta), \chi_{1}(z, \zeta)\right)+\tilde{d}\left((z, \zeta), \chi_{2}(x, \xi)\right) \gtrsim \tilde{d}\left((y, \eta), \chi_{1} \circ \chi_{2}(x, \xi)\right)
$$

Hence, using also the fact that $m_{1}$ is slowly varying we get

$$
\begin{gathered}
|K(y, \eta, x, \xi)| \lesssim m_{1}\left(\chi_{2}(x, \xi)\right) m_{2}(x, \xi) e^{-c N \tilde{d}\left((y, \eta), \chi_{1} \circ \chi_{2}(x, \xi)\right)} \\
\int e^{-c N \tilde{d}\left((z, \zeta), \chi_{2}(x, \xi)\right)} d z d \zeta
\end{gathered}
$$

which by Lemma 2.6 gives

$$
|K(y, \eta, x, \xi)| \lesssim m_{1}\left(\chi_{2}(x, \xi)\right) m_{2}(x, \xi) e^{-c N \tilde{d}\left((y, \eta),, \chi_{1} \circ \chi_{2}(x, \xi)\right)}
$$

Also we consider the boundedness of Fourier integral operators in $L^{2}$ and in Sobolev spaces.

Theorem 5.5. Let $\chi$ be a g-bi-Lipschitz canonical transformation and $A \in F I O(1, g, \chi)$. Then

$$
A: L^{2} \rightarrow L^{2}
$$

Proof. It suffices to show that $T A T^{*}$ is $L^{2}$ bounded. Its kernel $K$ satisfies

$$
|K(y, \eta, x, \xi)| \lesssim e^{-N \tilde{d}((y, \eta), \chi(x, \xi)))}
$$

Then by Lemma 2.6 we have

$$
\sup _{x, \xi} \int|K(y, \eta, x, \xi)| d y d \eta<\infty
$$

Since $\chi$ is bi-Lipschitz we also have

$$
|K(y, \eta, x, \xi)| \lesssim e^{\left.-N \tilde{d}\left(\chi^{-1}(y, \eta),(x, \xi)\right)\right)}
$$


which yields

$$
\sup _{y, \eta} \int|K(y, \eta, x, \xi)| d x d \xi<\infty
$$

This concludes the proof.

\section{Evolution EQUATIONS}

Here we study evolution equations of the form

$$
\left(D_{t}+a(t, x, D)\right) u=0 \quad u(0)=u_{0}
$$

where $t \in[0,1]$ and $x \in \mathbb{R}^{n}$. We assume that

(i) The symbol $a(t, x, \xi)$ is real.

(ii) $a_{x}$ is almost homogeneous,

$$
\left|a_{x}(t, x, \xi)\right| \lesssim\langle\xi\rangle, \quad\left|\xi \wedge\left(a_{x \xi}(t, x, \xi) \xi-a_{x}(x, \xi)\right)\right| \lesssim\langle\xi\rangle^{\frac{3}{2}}
$$

(iii) a satisfies (13), (17) uniformly in $t$. It also satisfies (12) for $\alpha \geq 2$ with one exception described in (ii).

As we prove later on, under these conditions the evolution (29) is $L^{2}$ well-posed. We denote by $S(t, s)$ the generated $L^{2}$ bounded evolution operator. Our goal is to describe $S(t, s)$ as Fourier integral operators associated to the corresponding Hamilton flow.

The Hamilton flow associated to this evolution is

$$
\dot{x}=a_{\xi}(t, x, \xi), \quad \dot{\xi}=-a_{x}(t, x, \xi)
$$

We denote the trajectories of the flow by

$$
[0,1] \ni t \rightarrow\left(x^{t}, \xi^{t}\right)
$$

and the fixed time maps by $\chi(t, s): T^{*} \mathbb{R}^{n} \rightarrow T^{*} \mathbb{R}^{n}$,

$$
\chi(t, s)\left(x^{s}, \xi^{s}\right)=\left(x^{t}, \xi^{t}\right)
$$

Then we have

Proposition 6.1. Under the assumptions (i), (ii), (iii) above the fixed time maps $\chi(t, s)$ are $g$-smooth g-bi-Lipschitz canonical transformations.

Proof. The fact that $\chi(t, s)$ is a canonical transformation is a property shared by all Hamiltonian flows. The bi-Lipschitz regularity is obtained by studying the linearized flow, which involves the second derivatives of the symbol $a$ :

$$
\begin{aligned}
& \dot{y}=a_{\xi x}\left(t, x^{t}, \xi^{t}\right) y+a_{\xi \xi}\left(t, x^{t}, \xi^{t}\right) \eta \\
& \dot{\eta}=-a_{x x}\left(t, x^{t}, \xi^{t}\right) y-a_{x \xi}\left(t, x^{t}, \xi^{t}\right) \eta
\end{aligned}
$$


For this we seek to propagate the norm

$$
g_{\xi^{t}}^{-1}(y)+g_{\xi^{t}}(\eta)
$$

via a Gronwall type inequality. We consider the two components in each of the two terms. By (iii) we have

$$
\left|a_{\xi x}\left(t, x^{t}, \xi^{t}\right) y\right| \lesssim\left\langle\xi^{t}\right\rangle^{-\frac{1}{2}} g_{\xi^{t}}^{-1}(y)^{\frac{1}{2}}, \quad\left|a_{\xi \xi}\left(t, x^{t}, \xi^{t}\right) \eta\right| \lesssim\left\langle\xi^{t}\right\rangle^{-\frac{1}{2}} g_{\xi^{t}}(\eta)^{\frac{1}{2}}
$$

hence the Euclidean length of $y$ is easy to propagate. We need a more refined computation for the component in the $\xi$ direction:

$$
\frac{d}{d t}\left(y \xi^{t}\right)=\xi^{t} a_{\xi x} y+\xi^{t} a_{\xi \xi} \eta-y a_{x}
$$

For the middle term we use again the condition (iii), but the first and the last are paired and we use (ii).

For $\eta$ the roles are reversed. It is easy to bound the component in the $\xi^{t}$ direction, but we need a better estimate for the component which is normal to $\xi^{t}$. We compute

$$
\frac{d}{d t}\left(\xi^{t} \wedge \eta\right)=-\xi^{t} \wedge a_{x x} y-\xi^{t} \wedge a_{x \xi} \eta-a_{x} \wedge \eta
$$

We use (iii) for the first term, and also in the last two but only for the component of $\eta$ which is normal to $\xi$. However, for the component of $\eta$ in the $\xi$ direction we use (ii).

Finally, to prove that $\chi$ is $g$-smooth one needs to further differentiate the linearized flow and use the relations (12) for $\alpha \geq 3$. This is a routine computation which is left for the reader.

Our main result is

Theorem 6.2. Assume that (i),(ii) and (iii) above hold. Then the evolution (29) is $L^{2}$ well-posed. Furthermore, the evolution operators $S(t, s)$ are Fourier integral operators associated to the Hamilton flow,

$$
S(t, s) \in F I O(1, g, \chi(t, s))
$$

Proof. We begin with a preliminary result.

Lemma 6.3. The function

$$
\psi_{\xi}(\eta)=\left(|\xi| \partial_{\xi}+|\eta| \partial_{\eta}\right) \phi_{\xi}(\eta)
$$

satisfies the same bounds as $\phi_{\xi}(\eta)$,

$$
\left|\nabla_{\xi}^{\alpha} \psi_{\xi}(\eta)\right|_{g} \leq c_{\alpha}
$$

Proof. The proof is routine but not totally trivial. 
Next we compute the action of pseudodifferential operators on coherent states.

Lemma 6.4. We have

$$
\begin{aligned}
A(t, x, D) \phi_{y, \eta}= & \left(a(t, y, \eta)-i\left(a_{x}(t, y, \eta) \partial_{\eta}-a_{\xi}(t, y, \eta)\left(\partial_{y}+i \eta\right)\right) \phi_{y, \eta}\right. \\
& +r_{y, \eta}
\end{aligned}
$$

where the remainder $r$ satisfies

$$
\left\langle r_{y, \eta}, \phi_{z, \zeta}\right\rangle=O\left(e^{-N d((y, \eta),(z, \zeta))}\right)
$$

Proof. The variable $t$ is irrelevant here so we drop it. We expand $a$ in a modified Taylor series around $(y, \eta)$,

$$
a(x, \xi)=a(y, \eta)+a_{\xi}(y, \eta)(\xi-\eta)+a_{x}(y, \xi)(x-y)+b(x, \xi)
$$

At the operator level this becomes

We apply this to $\phi_{y, \eta}$ and use the expression (7) to evaluate each of the terms. For the first term we need to do nothing. The Fourier transform of the second term is

$$
\mathcal{F}\left(a_{\xi}(y, \eta)\left(D_{x}-\eta\right) \phi_{y, \eta}\right)=i a_{\xi}(y, \eta)\left(\partial_{y}+i \eta\right) \hat{\phi}_{y, \eta}(\xi)
$$

For the third term we compute

$$
\begin{aligned}
& \mathcal{F}\left((x-y) a_{x}(y, D) \phi_{y, \eta}\right)=-\left(D_{\xi}+y\right)\left[a_{x}(y, \xi) \hat{\phi}_{y, \eta}(\xi)\right] \\
&=e^{-i y \xi} a_{x}(y, \xi) D_{\xi} \phi_{\eta}(\xi)+i a_{x \xi}(y, \xi) \hat{\phi}_{y, \eta}(\xi) \\
&=e^{-i y \xi} a_{x}(y, \eta) D_{\eta} \phi_{\eta}(\xi) \\
&+ e^{-i y \xi}\left(\langle\xi\rangle^{-1} a_{x}(y, \xi)-\langle\eta\rangle^{-1} a_{x}(y, \eta)\right)\langle\xi\rangle D_{\xi} \phi_{\eta}(\xi) \\
&++e^{-i y \xi}\langle\eta\rangle^{-1} a_{x}(y, \eta)\left(\langle\xi\rangle D_{\xi}-\langle\eta\rangle D_{\eta}\right) \psi_{\eta}(\xi) \\
&+i a_{x \xi}(y, \xi) \hat{\phi}_{y, \eta}(\xi)
\end{aligned}
$$

The first term is what we want, everything else must go into $r$. The second term is controlled due to the almost homogeneity of $a_{x}(x, \xi)$ in (ii). For the third term we use Lemma 6.3. For the fourth we use (iii).

Finally we consider the last term in (30). We claim that the symbol $B$ satisfies

$$
b \in \tilde{\tilde{S}}(m, g), \quad m=e^{N_{0} \tilde{d}(x, \xi ; y, \eta)}
$$

By Theorem 4.14 this shows that its contribution can be included in $r_{y, \eta}$. Derivatives of $b$ of order two and higher can be estimated directly using (ii) and (iii). The conditions (13) and (17) also follow trivially from the similar conditions for $a$. Hence it suffices to verify that

$$
|b(x, \xi)| \lesssim e^{N \tilde{d}(x, \xi ; y, \eta)}, \quad\left|\nabla_{g} b(x, \xi)\right| \lesssim e^{N \tilde{d}(x, \xi ; y, \eta)}
$$


The first bound follows from the second by integration along a geodesic. For the second we integrate the second derivative along a geodesic. This works well within balls of size one, but since $g$ is changing we loose a fixed factor when we move from a ball to the next one. This gives the above bound with $\tilde{d}$ replaced by $d$. To account for the change to $\tilde{d}$ we need to consider geodesics on the set $\xi=0$. There the metric is Euclidean, so integration only gives linear rather than exponential growth.

This allows us to conjugate the operator $A$ with respect to $T^{*}$. We define the selfadjoint phase space operator

$$
\tilde{A}=\left(a(t, y, \eta)+i\left(a_{x}(t, y, \eta) \partial_{\eta}-a_{\xi}(t, y, \eta)\left(\partial_{y}-i \eta\right)\right) \phi_{y, \eta} .\right.
$$

Then

Lemma 6.5. The operator $\tilde{A}$ is an approximate conjugate of $A$ with respect to $T^{*}$,

$$
A T^{*}-T^{*} \tilde{A}=R, \quad R T \in O P \tilde{\tilde{S}}(1, g)
$$

Proof. We have

$$
R f(x)=\int f\left(x_{2}, \xi_{2}\right) A(x, D) \phi_{x_{2}, \xi_{2}}-\phi_{x_{2}, \xi_{2}}(\tilde{A} f)\left(x_{2}, \xi_{2}\right) d x_{2} d \xi_{2}
$$

In the second term we integrate by parts to move the derivatives on $\phi_{x_{2}, \xi_{2}}$. By Lemma 6.4 this yields

$$
R f(x)=\int f\left(x_{2}, \xi_{2}\right) r_{x_{2}, \xi_{2}}(x) d x_{2} d \xi_{2}
$$

Then the kernel $K$ of $T R$ is

$$
K\left(\left(x_{1}, \xi_{1}\right),\left(x_{2}, \xi_{2}\right)\right)=\left\langle r_{x_{2}, \xi_{2}}, \phi_{x_{1}, \xi_{1}}\right\rangle
$$

which is rapidly decreasing off diagonal. To show that $R T \in O P \tilde{\tilde{S}}(1, g)$ we need to consider the kernel of $T R T T^{*}$. The kernel of $T T^{*}$ decreases rapidly off diagonal, hence so does the kernel of the composition.

Since $\tilde{A}$ is selfadjoint we write

$$
A-A^{*}=A T^{*} T-T^{*} T A^{*}=T^{*} \tilde{A} T+R T-T^{*} \tilde{A} T-T^{*} R^{*}=R T-T^{*} R^{*}
$$

Thus a consequence of this Lemma is that $A$ is essentially selfadjoint. 
Corollary 6.6. We have

$$
A-A^{*} \in O P \tilde{\tilde{S}}(1, g)
$$

This implies that the evolution (29) is $L^{2}$ well-posed, i.e. that the evolution operators $S(t, s)$ are $L^{2}$ bounded. Now we show that they are FIO's associated to the canonical transformations $\chi(t, s)$. Without any restriction in generality we take $s=0$. We need to obtain bounds for the kernel $K_{t}$ of $T S(t, 0) T^{*}$. More generally, given a solution $u=$ $S(t, 0) u_{0}$ to $(29)$ we seek to control the flow for $T u$.

By Lemma 6.5 and Corollary 6.6 we can write

$$
0=T\left(D_{t}+A(x, D)\right) u=\left(D_{t}+\tilde{A}\right) T u+R_{0}(t) u
$$

where $R_{0}(t) T^{*}$ has a kernel with rapid decay off the diagonal.

Hence if $u$ solves (29) then $v=T u$ solves

$$
\left(D_{t}+\tilde{A}\right) v=R_{1}(t) v
$$

which is a transport equation modulo a good integral part. We pull this back to time 0 using the $\tilde{A}$ flow $\tilde{S}(t, s)$,

$$
w(t)=\tilde{S}(0, t) v(t)
$$

This solves

$$
D_{t} w=R_{2}(t) w, \quad R_{2}(t)=\tilde{S}(0, t) R_{1}(t) \tilde{S}(t, 0)
$$

The pull back $R_{2}$ of $R_{1}$ still has a kernel with rapid off diagonal decay since $\tilde{S}(t, 0)$ corresponds to the transport along the Hamilton flow which is symplectic therefore measure preserving, and it also is $\tilde{d}$ biLipschitz.

Finally, to get bounds for $w$ we take absolute values above

$$
\partial_{t}|w(t, x, \xi)| \leq c_{M} \int|w(t, y, \eta)| e^{-M \tilde{d}((x, \xi),(y, \eta))} d y d \eta
$$

and use the maximum principle for $|w|$.

Precisely, in order to obtain bounds for the kernel of $T S(t, 0) T^{*}$ we take initial data $u_{0}=\phi_{x_{2}, \xi_{2}}$ and we want to prove that

$$
\left|v\left(t, x_{1}, \xi_{1}\right)\right| \leq c_{N} e^{-N \tilde{d}\left(\left(x_{1}, \xi_{1}\right), \chi(t, 0)\left(x_{2}, \xi_{2}\right)\right)}
$$

This translates to

$$
|w(t, x, \xi)| \leq c_{N} e^{-N \tilde{d}\left((x, \xi),\left(x_{2}, \xi_{2}\right)\right)}
$$

The initial data for $w$ is

$$
w(0)=T \phi_{x_{2}, \xi_{2}}
$$


therefore satisfies the above inequality with some constant $c_{N}(0)$. We claim that if $C$ is sufficiently large depending on $c_{2 N}$ in (31) then

$$
|w(t, x, \xi)| \leq e^{C t} c_{N}(0) e^{-N \tilde{d}\left((x, \xi),\left(x_{2}, \xi_{2}\right)\right)}
$$

For this we verify that the right hand side is a supersolution for (31) with $M=2 N$. We need to verify that

$$
C e^{-N \tilde{d}\left((x, \xi),\left(x_{2}, \xi_{2}\right)\right)} \geq C_{M} \int e^{-2 N \tilde{d}((x, \xi),(y, \eta))} e^{-N \tilde{d}\left((y, \eta),\left(x_{2}, \xi_{2}\right)\right)} d y d \eta
$$

Indeed from the triangle inequality we can bound the right hand side integrand by

$$
e^{-N \tilde{d}((x, \xi),(y, \eta))} e^{-N \tilde{d}\left((x, \xi),\left(x_{2}, \xi_{2}\right)\right)}
$$

and then use Lemma (2.6) to carry out the integration.

Let $B(0)$ be a pseudodifferential operator. Conjugating it with respect to the $D_{t}+A$ flow we obtain a time dependent family of operators

$$
B(t)=S(t, 0) B(0) S(0, t)
$$

Given a slowly varying weight $m$ with respect to the metric $g$, the composition result in Theorem 5.4 yields

Proposition 6.7. Assume that $B(0) \in O P \tilde{\tilde{S}}(m, g)$. Then for all $t$ we have $B(t) \in O P \tilde{\tilde{S}}(m \circ \chi(t, 0), g)$.

We would like to obtain an Egorov type result, i.e. to characterize the symbol of $B(t)$ in terms of the symbol of $B(0)$. In the context of the above result this is not possible since the metric $g$ is exactly on the scale of the uncertainty principle. However, we can still prove a result which amounts to a first order calculus:

Theorem 6.8. Assume that a satisfies (i),(ii) and (iii). Let $m$ be a slowly varying weight and $B(0)$ be a pseudodifferential operator whose symbol satisfies (12) for all $|\alpha| \geq 1$, and also (13), (17). Then $B(t)$ is a pseudodifferential operator which belongs to the same class. In addition,

$$
b(t)-b(0) \circ \chi(0, t) \in S(m, g)
$$

Proof. For fixed $\left(x_{0}, \xi_{0}\right)$ we define the operators

$$
C(t)=B(t)-b\left(0, x_{0}, \xi_{0}\right)
$$

Then $c\left(0, x_{0}, \xi_{0}\right)=0$. Hence, using (12) for $b(0)$ with $|\alpha|=1$ we obtain

$$
|c(0, x, \xi)| \lesssim m_{x_{0}, \xi_{0}}(x, \xi):=m(x, \xi) e^{C \tilde{d}\left(x, \xi, x_{0}, \xi_{0}\right)}
$$


Thus we have

$$
c(0) \in \tilde{\widetilde{S}}\left(m_{x_{0}, \xi_{0}}, g\right)
$$

By the previous proposition, this yields

$$
c(t) \in \tilde{\tilde{S}}\left(m_{x_{0}, \xi_{0}} \circ \chi(t, 0), g\right)
$$

Using (12) for $c(t)$ in the unit ball centered at $\left(x_{0}, \xi_{0}\right)$ we conclude that

$$
\left|b\left(t, x_{0}^{t}, \xi_{0}^{t}\right)-b\left(0, x_{0}, \xi_{0}\right)\right| \lesssim m\left(x_{0}, \xi_{0}\right)
$$

and also that $b(t)$ satisfies (12) for $|\alpha| \geq 1$ with respect to the weight $m \circ \chi(0, t)$.

The conditions (13), (17) for $b(t)$ follow directly from the similar conditions for $c(t)$ with $(x, \xi)=\left(x_{0}, \xi_{0}\right)$.

\section{HALF-WAVE OPERATORS AND PARADIFFERENTIAL CALCULUS}

A large class of symbols which satisfy the conditions of the previous section can be obtained from half wave operators whose coefficients are mollified in a paradifferential fashion. Precisely, we begin with a real symbol $a(x, \xi)$ which is homogeneous of order 1 in $\xi$, and satisfies the following regularity conditions:

(a) $a(x, \xi)$ is smooth in $\xi$.

(b) $\left|\nabla_{x} a(x, \xi)\right| \lesssim|\xi|$

(c) $\left|\left(\xi \wedge \nabla_{x}\right)^{2} a(x, \xi)\right| \lesssim|\xi|^{3}$.

Then we consider the evolution governed by $a(x, D)$,

$$
\left(D_{t}+A(x, D)\right) u=0, \quad u(0)=u_{0}
$$

The Hamilton flow

$$
\left\{\begin{array}{c}
\dot{x}=a_{\xi}(x, \xi) \\
\dot{\xi}=-a_{x}(x, \xi)
\end{array}\right.
$$

is homogeneous. To describe its regularity we introduce a subelliptic Riemannian metric $g_{\text {hom }}$ on the cosphere bundle

$$
S^{*} \mathbb{R}^{n}=\mathbb{R}^{n} \times S^{n-1}
$$

If we denote by $(x, \xi)$ the coordinates in $S^{*} \mathbb{R}^{n}$ then $g_{\text {hom }}$ is defined by

$$
d s^{2}= \begin{cases}d x^{2}+d \xi^{2} & \text { if } d x \cdot \xi=0 \\ \infty & \text { otherwise }\end{cases}
$$

We note that at each $(x, \xi)$ this metric only allows for displacements in directions which are perpendicular to $(\xi, 0)$.

We denote by $d_{\text {hom }}$ the induced distance. The following characterization shows that this is is related to Smith's pseudometric in [12], $[11]$. 
Lemma 7.1. We have

$$
d_{\text {hom }}\left(\left(x_{0}, \xi_{0}\right),\left(x_{1}, \xi_{1}\right)\right) \approx\left|x_{0}-x_{1}\right|+\left|\xi_{0}-\xi_{1}\right|+\left|\left(x_{0}-x_{1}\right) \xi_{0}\right|^{\frac{1}{2}}
$$

Proof. We first prove the inequality

$$
\left|x_{0}-x_{1}\right|+\left|\xi_{0}-\xi_{1}\right|+\left|\left(x_{0}-x_{1}\right) \xi_{0}\right|^{\frac{1}{2}} \lesssim d_{\text {hom }}\left(\left(x_{0}, \xi_{0}\right),\left(x_{1}, \xi_{1}\right)\right)
$$

The first two terms on the left are easy to bound, it remains to consider the third. Let $\gamma$ be an admissible trajectory between $\left(x_{0}, \xi_{0}\right)$ and $\left(x_{1}, \xi_{1}\right)$. Since $\xi d x=0$ on $\gamma$ we write

$$
\left(x_{0}-x_{1}\right) \xi_{0}=\int_{\gamma} \xi_{0} d x=\int_{\gamma}\left(\xi_{0}-\xi\right) d x
$$

Taking absolute values,

$$
\left|\left(x_{0}-x_{1}\right) \xi_{0}\right| \leq \int_{\gamma}\left|\xi_{0}-\xi\right| d s \leq \int_{\gamma}|\gamma| d s=|\gamma|^{2}
$$

It remains to prove the converse, i.e. to find an a trajectory $\gamma$ between $\left(x_{0}, \xi_{0}\right)$ and $\left(x_{1}, \xi_{1}\right)$ whose length is at most comparable to the right hand side in (32). We first simplify the problem somewhat. By moving first from $\left(x_{0}, \xi_{0}\right)$ to $\left(x_{1}, \xi_{0}\right)$ and then along the spherical geodesic from $\left(x_{1}, \xi_{0}\right)$ to $\left(x_{1}, \xi_{1}\right)$ we reduce the problem to the case $\xi_{0}=\xi_{1}$. In a similar fashion we dispense with the component of $x_{0}-x_{1}$ which is perpendicular to $\xi_{0}$.

Thus we have reduced the problem to estimating $d\left(\left(0, \xi_{0}\right),\left(\epsilon \xi_{0}, \xi_{0}\right)\right)$. It suffices to work in two dimensions, where we can use complex notations.

If $\epsilon>1$ then we move from $\left(0, \xi_{0}\right)$ to $\left(0, i \xi_{0}\right)$, then straight to $\left(\epsilon \xi_{0}, i \xi_{0}\right)$ and back to $\left(\epsilon \xi_{0}, \xi_{0}\right)$ for an $O(1+\epsilon)$ distance.

If $\epsilon<1$ then we choose a trajectory composed of straight lines and spherical geodesics as follows:

$$
\begin{aligned}
\left(0, \xi_{0}\right) & \rightarrow\left(0, e^{i \theta} \xi_{0}\right) \rightarrow\left(\frac{i \epsilon e^{i \theta}}{2 \sin \theta} \xi_{0}, e^{i \theta} \xi_{0}\right) \rightarrow\left(\frac{i \epsilon e^{i \theta}}{2 \sin \theta} \xi_{0}, e^{-i \theta} \xi_{0}\right) \\
& \rightarrow\left(\epsilon \xi_{0}, e^{-i \theta} \xi_{0}\right) \rightarrow\left(\epsilon \xi_{0}, \xi_{0}\right)
\end{aligned}
$$

Its length is $O(|\theta|+\epsilon /|\theta|)$. The optimal balance is reached at $\theta \approx \sqrt{\epsilon}$ for a length of $O(\sqrt{\epsilon})$.

Next we study the regularity of the Hamilton flow of $a$ using this metric.

Proposition 7.2. Assume that a satisfies (a),(b) and (c). Then its Hamilton flow maps $\chi(t, s)$ are homogeneous and $g_{\text {hom }}$-Lipschitz. 
Proof. Consider $\gamma_{0}(t)=\left(x_{0}(t), \xi_{0}(t)\right)$ respectively $\gamma_{1}(t)=\left(x_{1}(t), \xi_{1}(t)\right)$ two trajectories of the Hamilton flow in $[0, T]$. We take $\epsilon>0$ and $T$ small and prove that

$$
d_{\text {hom }}\left(\gamma_{0}(0), \gamma_{1}(0)\right)<\epsilon \Rightarrow d_{\text {hom }}\left(\gamma_{0}(t), \gamma_{1}(t)\right)<C \epsilon
$$

Without any restriction in generality we can make the bootstrap assumption

$$
d_{\text {hom }}\left(\gamma_{0}(t), \gamma_{1}(t)\right)<2 C \epsilon
$$

which we rewrite in an expanded fashion as

$$
\begin{gathered}
\left|x_{0}(t)-x_{1}(t)\right| \lesssim \epsilon, \quad\left|\left(x_{0}(t)-x_{1}(t)\right) \xi_{0}(t)\right| \lesssim \epsilon^{2}\left|\xi_{0}(t)\right| \\
\left|\frac{\xi_{0}(t)}{\left|\xi_{0}(t)\right|}-\frac{\xi_{1}(t)}{\left|\xi_{1}(t)\right|}\right| \lesssim \epsilon
\end{gathered}
$$

To propagate the first relation we need to show that

$$
\left|a_{\xi}\left(x_{0}(t), \xi_{0}(t)\right)-a_{\xi}\left(x_{1}(t), \xi_{1}(t)\right)\right| \lesssim \epsilon
$$

which follows since $a_{\xi}$ is Lipschitz continuous.

For the second component we compute

$$
\begin{aligned}
\frac{d}{d t}\left[\left(x_{0}(t)-x_{1}(t)\right) \xi_{0}(t)\right]= & \left(a_{\xi}\left(x_{0}(t), \xi_{0}(t)\right)-a_{\xi}\left(x_{1}(t), \xi_{1}(t)\right)\right) \xi_{0}(t) \\
& -\left(x_{0}(t)-x_{1}(t)\right) a_{x}\left(x_{0}(t), \xi_{0}(t)\right)
\end{aligned}
$$

Since $a$ is smooth in $\xi$ we can rewrite this as

$$
\begin{aligned}
\frac{d}{d t}\left[\left(x_{0}(t)-x_{1}(t)\right) \xi_{0}(t)\right]= & \left(a_{\xi}\left(x_{0}(t), \xi_{0}(t)\right)-a_{\xi}\left(x_{1}(t), \xi_{0}(t)\right)\right) \xi_{0}(t) \\
& \left.+\left(\xi_{1}(t)\right)-\xi_{0}(t)\right) a_{\xi \xi}\left(x_{1}(t), \xi_{0}(t)\right) \xi_{0}(t) \\
& -\left(x_{0}(t)-x_{1}(t)\right) a_{x}\left(x_{0}(t), \xi_{0}(t)\right)+O\left(\epsilon^{2}\right)\left|\xi_{0}\right|
\end{aligned}
$$

By homogeneity the second term drops and the first one simplifies to give

$\left.a\left(x_{0}(t), \xi_{0}(t)\right)-a\left(x_{1}(t), \xi_{0}(t)\right)\right)-\left(x_{0}(t)-x_{1}(t)\right) a_{x}\left(x_{0}(t), \xi_{0}(t)\right)+O\left(\epsilon^{2}\right)\left|\xi_{0}\right|$

The component of $x_{1}-x_{0}$ which is perpendicular to $\xi_{0}$ has size $O\left(\epsilon^{2}\right)$ which is acceptable. For the parallel component on the other hand we use the Taylor expansion of second order to gain the $O\left(\epsilon^{2}\right)$.

Finally to propagate the third relation in (33) we need to show that

$$
\left.\left.\left.|| \xi_{0}(t)\right|^{-1} \Pi_{\xi_{0}(t)} a_{x}\left(x_{0}(t)\right), \xi_{0}(t)\right)-\left|\xi_{1}(t)\right|^{-1} \Pi_{\xi_{1}(t)} a_{x}\left(x_{1}(t)\right), \xi_{1}(t)\right) \mid \lesssim \epsilon
$$

Since $a_{x}$ is Lipschitz in $\xi$ we can replace $\xi_{1}$ by $\xi_{0}$ in the second term modulo $O(\epsilon)$ errors. It remains to show that

$$
\left.\left.\mid \eta\left(a_{x}\left(x_{0}(t)\right), \xi_{0}(t)\right)-a_{x}\left(x_{1}(t)\right), \xi_{0}(t)\right)\right)|\lesssim \epsilon| \xi_{0}(t) \mid
$$


where $\eta$ is a unit vector normal to $\xi_{0}$. As a function of $x$ the function $\eta\left(a_{x}\left(x, \xi_{0}(t)\right)\right.$ is Lipschitz in directions perpendicular to $\xi_{0}$ and Hölder $1 / 2$ in the $\xi_{0}$ direction. This suffices for the desired conclusion.

One can easily prove that $A-A^{*}$ is $L^{2}$ bounded, which implies that the above evolution is well-posed in $L^{2}$. However, due to the low regularity of the coefficients there is considerable interaction between different frequencies. Thus in order to better understand the flow it is convenient to replace the operator $A$ with an $L^{2}$ bounded perturbation of it, obtained by regularizing the symbol in $x$. Precisely, we define the modified symbol

$$
\tilde{a}(x, \xi)=S_{\xi}\left(D_{x}\right) a(x, \xi)
$$

where the symbol of $S_{\xi}$ is given by

$$
S_{\xi}(\eta)=h\left(g_{\xi}(\eta)\right)
$$

The evolution generated by $\tilde{A}$ can be described using Fourier integral operators as in the previous section provided that

Proposition 7.3. If the symbol a satisfies (a),(b) and (c) then the symbol $\tilde{a}$ satisfies the conditions (i),(ii),(iii) in Section 6.

To see that the evolution generated by $A$ is a Lipschitz perturbation of the evolution generated by $\tilde{A}$ we need

Proposition 7.4. If the symbol a satisfies (a),(b) and (c) then the difference $A(x, D)-\tilde{A}(x, D)$ is $L^{2}$ bounded.

Finally, the Hamilton flow for $\tilde{a}$ stays close to the Hamilton flow for $a$, so the two canonical transformations are interchangeable in the definition of Fourier integral operators. Denoting by $\tilde{\chi}(t, s)$ the Hamilton flow maps for $\tilde{a}$ we have

Proposition 7.5. If the symbol a satisfies (a),(b) and (c) then the Hamilton flows for a and $\tilde{a}$ are close,

$$
d(\chi(t, s)(x, \xi), \tilde{\chi}(t, s)(x, \xi)) \lesssim 1
$$

Proof of Proposition 7.3. The bound $\left|a_{x}\right| \lesssim|\xi|$ easily leads to $\left|\tilde{a}_{x}\right| \lesssim \xi$. For the almost homogeneity of $\tilde{a}$ we compute

$$
\begin{aligned}
\xi \partial_{\xi} \tilde{a}_{x}(x, \xi) & =\left(\xi \partial_{\xi} S_{\xi}\left(D_{x}\right)\right) a(x, \xi)+S_{\xi}\left(D_{x}\right) \xi \partial_{\xi} a(x, \xi) \\
& =\left(\xi \partial_{\xi} S_{\xi}\left(D_{x}\right)\right) a(x, \xi)+S_{\xi}\left(D_{x}\right) a(x, \xi) \\
& =\left(\xi \partial_{\xi} S_{\xi}\left(D_{x}\right)\right) a(x, \xi)+\tilde{a}_{x}(x, \xi)
\end{aligned}
$$


Finally we consider the regularity of the derivatives of $\tilde{a}$ of second order and higher,

$$
\nabla_{g_{\xi}^{-1}}^{\alpha} \nabla_{g_{\xi}}^{\beta} \tilde{a}(x, \xi)=\nabla_{g_{\xi}^{-1}}^{\alpha} \nabla_{g_{\xi}}^{\beta}\left(S_{\xi}\left(D_{x}\right) a(x, \xi)\right)
$$

We first argue why we gain a $|\xi|^{-1}$ factor. In the case of $x$ derivatives, we can put onto $a$ either one derivative in the $\xi$ direction or two derivatives perpendicular to $\xi$. In both cases we gain $|\xi|^{-1}$.

In the case of $\xi$ derivatives we consider three cases. If one $\xi$ derivative falls on $S_{\xi}$ then we obtain a similar symbol $\tilde{S}_{\xi}$ but which in addition is supported away from the origin. Then we use the bound

$$
\left|\tilde{S}_{\xi}(D) a(x)\right| \lesssim|\xi|^{-1}\left(\left\||\xi|^{-1} \xi \nabla a\right\|_{L^{\infty}}+\left\||\xi|^{-2}(\xi \wedge \nabla)^{2} a\right\|_{L^{\infty}}\right)
$$

If we have two derivatives perpendicular to $\xi$ on $a(x, \xi)$ then we gain $|\xi|^{-1}$ because of the regularity of $a$. If we have two derivatives of which at least one is in the $\xi$ direction then we get 0 because of the homogeneity of $a$.

Consider now the mixed case. If we have one $x$ derivative and one $\xi$ derivative on $a$ both in directions perpendicular to $\xi$ then we gain $|\xi|^{-\frac{1}{2}}$ from each. If instead the $\xi$ derivative is in the $\xi$ direction, this is precisely the one case we do not need.

Additional $\xi$ derivatives which fall on $a$ are well behaved since $a$ is smooth and homogeneous in $\xi$. The ones which fall on $S_{\xi}$ on the other hand yield symbols of similar size and support. Additional $x$ derivatives have a similar effect on the symbol of $S_{\xi}$.

Proof of Proposition 7.4. We introduce an intermediate weaker symbol regularization,

$$
\tilde{a}_{0}(x, \xi)=S_{\langle\xi\rangle}\left(D_{x}\right) a(x, \xi)
$$

where the symbol of $S_{\langle\xi\rangle}\left(D_{x}\right)$ is

$$
S_{\langle\xi\rangle}(\eta)=h\left(\frac{\langle\eta\rangle}{4\langle\xi\rangle}\right)
$$

First we bound in $L^{2}$ the difference $A(x, D)-\tilde{A}_{0}(x, D)$. For this we only use the $C^{1}$ spatial regularity of the symbol $A$. Expanding $a$ in spherical harmonics the problem reduces to the case when

$$
a(x, \xi)=a(x) b(\xi)
$$

For this we need to show that

$$
\|B(a, \nabla f)\|_{L^{2}} \lesssim\|a\|_{C^{1}}\|f\|_{L^{2}}
$$


where $B$ is the bilinear multiplier with symbol

$$
b(\eta, \xi)=1-h\left(\frac{\langle\eta\rangle}{4\langle\xi\rangle}\right)
$$

But this is a direct consequence of the Coifman-Meyer estimates [4].

Secondly, we bound in $L^{2}$ the difference $C(x, D)=\tilde{A}(x, D)-\tilde{A}_{0}(x, D)$. Its symbol

$$
c(x, \xi)=\left(S_{\xi}\left(D_{x}\right)-S_{\langle\xi\rangle}\left(D_{x}\right)\right) a(x, \xi)
$$

has spatial Fourier transform supported in

$$
\{4\langle\eta\rangle<\langle\xi\rangle\} \backslash\left\{8 g_{\xi}(\eta)>1\right\}
$$

The homogeneity of $a$ is not useful here. Instead we do a LittlewoodPaley decomposition in $\xi$,

$$
a(x, \xi)=\sum_{j=0}^{\infty} a_{j}(x, \xi), \quad \operatorname{supp} a_{j}(x, \xi) \subset\left\{\langle\xi\rangle \approx 2^{j}\right\}
$$

Correspondingly we have a decomposition

$$
c(x, \xi)=\sum c_{j}(x, \xi)
$$

Due to the above support condition, $c_{j}$ takes frequencies of size $2^{j}$ into frequencies of the same size. By orthogonality it suffices to show that $c_{j}(x, D)$ are $L^{2}$ bounded.

To fix the spatial and frequency scales we consider an additional decomposition for $c_{j}$,

$$
c_{j}(x, \xi)=\sum_{k=0}^{j} c_{j k}(x, D)
$$

where

$$
c_{j k}(x, \xi)=S_{\langle\xi\rangle}\left(D_{x}\right)\left(S_{2^{k+1} \xi}\left(D_{x}\right)-S_{2^{k} \xi}\left(D_{x}\right)\right) a_{j}(x, \xi)
$$

For small $k$, say $k \leq 8$, it is easy to see that

$$
c_{j k} \in \tilde{S}(1, g)
$$

which implies that they are $L^{2}$ bounded. For larger $k$ we note that the multiplier

$$
S_{\langle\xi\rangle}(\eta)\left(S_{2^{k+1} \xi}(\eta)-S_{2^{k} \xi}(\eta)\right)
$$

is supported in the region

$$
\left\{\langle\eta\rangle \ll 2^{j},|\langle\eta\rangle \wedge \xi| \approx 2^{j+\frac{j+k}{2}}\right\}
$$


Using the condition (c) on the regularity of the symbol $a$ we conclude that $c_{j k}$ satisfies the pointwise bound

$$
\left|c_{j k}(x, \xi)\right| \lesssim 2^{-k}
$$

To finish the proof of the lemma it suffices to show that we also have a similar $L^{2}$ bound,

$$
\left\|C_{j k}(x, D)\right\|_{L^{2} \rightarrow L^{2}} \lesssim 2^{-k}
$$

To prove this we split the region $\left\{\langle\xi\rangle \approx 2^{j}\right\}$ into angular tubes $R_{l}$ of size

$$
2^{j} \times\left(2^{\frac{k+j}{2}}\right)^{n-1}
$$

We make a further angular decomposition in $\xi$,

$$
c_{j k}(x, \xi)=\sum_{l} c_{j k}^{l}(x, \xi), \quad \operatorname{supp}_{\xi} c_{j k}^{l}(x, \xi) \subset R_{l}
$$

The symbols $c_{j k}^{l}$ satisfy (34), are smooth in $\xi$ on the scale of $R_{l}$ and in $x$ on the dual scale. Hence they satisfy

$$
\left\|C_{j k}^{l}(x, D)\right\|_{L^{2} \rightarrow L^{2}} \lesssim 2^{-k}
$$

In addition, the Fourier supports of their outputs are essentially disjoint. Thus (35) follows.

Proof of Proposition 7.5. We follow the same argument as in the proof of Proposition 7.2. We consider bicharacteristics $\left(x_{0}(t), \xi_{0}(t)\right)$ for $a$ and $\left(x_{1}(t), \xi_{1}(t)\right)$ for $\tilde{a}$ with the same initial data $(x, \xi)$ at time 0 . We assume that $|\xi| \approx \lambda$. Then it is easy to see that we must also have $\left.\mid \xi_{0}(t)\right)|\approx \lambda,| \xi_{1}(t) \mid \approx \lambda$. Hence we should prove that

$$
\begin{array}{r}
\left|x_{0}(t)-x_{1}(t)\right| \leq \lambda^{-\frac{1}{2}}, \quad\left|\left(x_{0}(t)-x_{1}(t)\right) \xi_{0}(t)\right| \leq 1 \\
\left|\frac{\xi_{0}(t)}{\left|\xi_{0}(t)\right|}-\frac{\xi_{1}(t)}{\left|\xi_{1}(t)\right|}\right| \leq \lambda^{-\frac{1}{2}}
\end{array}
$$

which is similar to the computation in part (a) with $\epsilon=\lambda^{-\frac{1}{2}}$. We make a bootstrap assumption as before by doubling the constants.

We seek to prove the three relations using the bootstrap assumption and the Hamilton flow equations. Modulo the computation in part (a), for the first term we need to show that

$$
\left|(\tilde{a}-a)_{\xi}\left(x_{1}(t), \xi_{1}(t)\right)\right| \lesssim \lambda^{-\frac{1}{2}}
$$

For the second we need

$$
\left|(\tilde{a}-a)_{\xi}\left(x_{1}(t), \xi_{1}(t)\right) \xi_{1}\right| \lesssim 1
$$


Finally for the third we want

$$
\left.\mid \Pi_{\xi_{1}(t)}(a-\tilde{a})_{x}\left(x_{1}(t)\right), \xi_{1}(t)\right) \mid \lesssim \lambda^{\frac{1}{2}}
$$

\section{REFERENCES}

1. Jean-Michel Bony, Calcul symbolique et propagation des singularités pour les équations aux dérivées partielles non linéaires, Ann. Sci. École Norm. Sup. (4) 14 (1981), no. 2, 209-246. MR MR631751 (84h:35177)

2. Evolution equations and microlocal analysis, Hyperbolic problems and related topics, Grad. Ser. Anal., Int. Press, Somerville, MA, 2003, pp. 17-40. MR MR2056840 (2005f:35348)

3. Emmanuel J. Candès and David L. Donoho, New tight frames of curvelets and optimal representations of objects with piecewise $C^{2}$ singularities, Comm. Pure Appl. Math. 57 (2004), no. 2, 219-266. MR MR2012649 (2004k:42052)

4. Ronald R. Coifman and Yves Meyer, Au delà des opérateurs pseudodifférentiels, Astérisque, vol. 57, Société Mathématique de France, Paris, 1978, With an English summary. MR MR518170 (81b:47061)

5. Antonio Córdoba and Charles Fefferman, Wave packets and Fourier integral operators, Comm. Partial Differential Equations 3 (1978), no. 11, 979-1005. MR MR507783 (80a:35117)

6. Gerald B. Folland, Harmonic analysis in phase space, Annals of Mathematics Studies, vol. 122, Princeton University Press, Princeton, NJ, 1989. MR MR983366 (92k:22017)

7. Lars Hörmander, The analysis of linear partial differential operators. III, Grundlehren der Mathematischen Wissenschaften [Fundamental Principles of Mathematical Sciences], vol. 274, Springer-Verlag, Berlin, 1985, Pseudodifferential operators. MR MR781536 (87d:35002a)

8. __ Pseudo-differential operators of type 1, 1, Comm. Partial Differential Equations 13 (1988), no. 9, 1085-1111. MR MR946283 (89k:35260)

9._ Lectures on nonlinear hyperbolic differential equations, Mathématiques \& Applications (Berlin) [Mathematics \& Applications], vol. 26, Springer-Verlag, Berlin, 1997. MR MR1466700 (98e:35103)

10. Herbert Koch and Daniel Tataru, Dispersive estimates for principally normal pseudodifferential operators, Comm. Pure Appl. Math. 58 (2005), no. 2, 217284. MR MR2094851

11. Hart F. Smith, A Hardy space for Fourier integral operators, J. Geom. Anal. 8 (1998), no. 4, 629-653. MR MR1724210 (2001f:35459)

12. __ A parametrix construction for wave equations with $C^{1,1}$ coefficients, Ann. Inst. Fourier (Grenoble) 48 (1998), no. 3, 797-835. MR MR1644105 (99h:35119)

13. Hart F. Smith and Daniel Tataru, Sharp local well-posedness results for the nonlinear wave equation, To appear in Ann. of Math.

14. Daniel Tataru, Phase space transforms and microlocal analysis, Phase space analysis of partial differential equations, Pisa 2004, pp. 505-524. 
A PHASE SPACE TRANSFORM ADAPTED TO THE WAVE EQUATION 37

15. - On the Fefferman-Phong inequality and related problems, Comm. Partial Differential Equations 27 (2002), no. 11-12, 2101-2138. MR MR1944027 (2003m:35259)

16. _ Null form estimates for second order hyperbolic operators with rough coefficients, Harmonic analysis at Mount Holyoke (South Hadley, MA, 2001), Contemp. Math., vol. 320, Amer. Math. Soc., Providence, RI, 2003, pp. 383409. MR MR1979953 (2004d:35177)

17. Daniel Tataru and Dan-Andrei Geba, Dispersive estimates for wave equations, Comm. Partial Differential Equations 30 (2005), no. 5-6, 849-880.

Department of Mathematics, Evans Hall, University of California At Berkeley, Berkeley, CA 94720-3840 